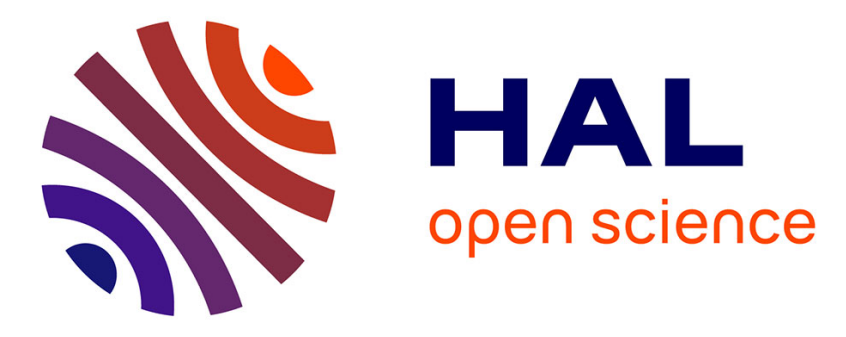

\title{
Multifractal Random Walks as Fractional Wiener Integrals
}

\author{
Patrice Abry, Pierre Chainais, Laure Coutin, Vladas Pipiras
}

\section{To cite this version:}

Patrice Abry, Pierre Chainais, Laure Coutin, Vladas Pipiras. Multifractal Random Walks as Fractional Wiener Integrals. IEEE Transactions on Information Theory, 2009, 55 (8), pp.3825-3846. 10.1109/TIT.2009.2023708 . hal-00808604

\section{HAL Id: hal-00808604 https://hal.science/hal-00808604}

Submitted on 9 Apr 2013

HAL is a multi-disciplinary open access archive for the deposit and dissemination of scientific research documents, whether they are published or not. The documents may come from teaching and research institutions in France or abroad, or from public or private research centers.
L'archive ouverte pluridisciplinaire HAL, est destinée au dépôt et à la diffusion de documents scientifiques de niveau recherche, publiés ou non, émanant des établissements d'enseignement et de recherche français ou étrangers, des laboratoires publics ou privés. 


\title{
Multifractal random walks as fractional Wiener integrals $* \dagger \ddagger$
}

\author{
Patrice Abry \\ ENS, Lyon \& CNRS \\ Pierre Chainais \\ Laure Coutin \\ Université Paul-Sabatier \\ Université Clermont-Ferrand \& CNRS \\ Vladas Pipiras \\ University of North Carolina
}

April 16, 2009

\begin{abstract}
Multifractal random walks are defined as integrals of infinitely divisible stationary multifractal cascades with respect to fractional Brownian motion. Their key properties are studied, such as finiteness of moments and scaling, with respect to the chosen values of the self-similarity and infinite divisibility parameters. The range of these parameters is larger than that considered previously in the literature, and the cases of both exact and non-exact scale invariance are considered. Special attention is paid to various types of definitions of multifractal random walks. The resulting random walks are of interest in modeling multifractal processes whose marginals exhibit stationarity and symmetry.
\end{abstract}

\section{Introduction}

Random multifractal measures and processes (or simply, multifractals) have been used to model natural and man-made phenomena in a variety of fields, ranging from turbulence in hydrodynamics, DNA sequences in genetics, rainfall in geophysics, to stock prices in finance, teletraffic in the Internet. Multifractals are described in at least two complementary ways. On one hand, the so-called multifractal spectrum functions are used to describe (e.g. Hausdorff) dimensions of singularity exponents of multifractals. On the other hand, the so-called partition functions are used to describe scaling properties of moments of multifractals. Multifractal formalism allows one to relate the two approaches (or the two sets of functions) through the Legendre transformation. See, for example, a review article by Riedi (2003) and references therein for more information on multifractals.

Much of the initial effort to construct random multifractals was directed to multifractal random measures which are nonnegative. Examples are the celebrated multiplicative (binomial) cascades of Mandelbrot (1974), Kahane and Peyriere (1976) or their more recent generalizations to compound Poisson cascades of Barral and Mandelbrot (2002), to (log-)infinitely divisible multifractal measures of Bacry and Muzy (2002, 2003), Schmitt and Marsan (2001), Schmitt (2003) and to non-scale invariant infinitely divisible cascades of Chainais, Riedi and Abry (2005a, 2005b). See also Mannersalo, Norros and Riedi (2002), Schertzer and Lovejoy (1987) for other constructions of multifractals. In many applications of multifractals, however, it is needed to have multifractal processes that take both

\footnotetext{
*The first author gratefully acknowledges the support of the Foundation Del Duca, Institut de France, 2007 grant. The last author was supported in part by the NSF grant DMS-0505628

${ }^{\dagger}$ This work was undertaken when V. Pipiras was visiting P. Abry and the Laboratoire de Physique at ENS, Lyon, under the support of a CNRS Visiting Researcher position. The author is grateful for their hospitality.

${ }^{\ddagger}$ Keywords and phrases: multifractals, multifractal random walks, fractional Brownian motion, fractional Wiener integrals, infinitely divisible cascades, scaling properties.
} 
positive and negative values, and that have stationary increments. Several models of multifractal processes were proposed to address this need.

A common way (see, for example, Mandelbrot (1999)) to construct a multifractal process is through

$$
Z(t)=Y(X([0, t])), \quad t \in \mathbb{R},
$$

where $X$ is a multifractal random measure and $Y$ is a self-similar process with stationary increments, independent of $X$. For more information on self-similar processes, see, for example, Section 7 in Samorodnitsky and Taqqu (1994), Embrechts and Maejima (2002). A typical choice for $Y$ is fractional Brownian motion $(\mathrm{fBm}) B_{H}, H \in(0,1)$, which is the only Gaussian $H$-self-similar process with stationary increments. In this case, the multifractal process (1.1) is also known as fBm in multifractal time, following Mandelbrot's terminology (cf. Mandelbrot (1999)). An advantage of working with processes (1.1) is that their multifractal properties are easy to deduce from those of $Y$ and $X$.

Another suggested way to construct a multifractal process is through stochastic integration as

$$
Z(t)=\int_{0}^{t} Q(u) d Y(u), \quad t \in \mathbb{R},
$$

where $Q$ is a suitable stationary nonnegative multifractal noise (for example, such as in Barral and Mandelbrot (2002)) and $Y$ is as in (1.1), independent of $Q$. Multifractal processes (1.2) are generally known as Multifractal Random Walks (MRWs, in short). The case of Brownian motion (Bm) $Y=B$ was studied and is quite easy to deal with because (1.2) is conditionally (on $Q$ ) Gaussian and the moments of $Z(t)$ can be expressed as

$$
E|Z(t)|^{q}=E|\mathcal{N}(0,1)|^{q} \cdot E\left(\int_{0}^{t} Q(u)^{2} d u\right)^{q / 2},
$$

where $E$ stands for the mathematical expectation (see, for example, p. 460 in Bacry and Muzy (2003)). Basic interests in introducing (1.2) are that their multifractal properties are generally different from those of (1.1) (thus contributing to the collection of available multifractal models), that their generating structure is quite different from (1.1) (thus offering potential alternative generating schemes of multifractal processes) and that their sample paths appear visually different from (1.1). In particular, regarding their generating structures, $Q(u)$ can be thought as determining instantaneous variance of a random system (1.2). Since $Q(u)$ is a multifractal noise, the variability fluctuates with bursts of activity associated with high values of $Q(u)$. On the other hand, the activity of a random system (1.1) is thought to be governed by a multifractal time $X(t)$. Sudden increases in $X(t)$ are now associated with faster activity of the system (1.1). (See also Figures 3 and 4 below, and Section 4.6.)

In this work, we study processes (1.2) with $\mathrm{fBm} Y=B_{H}$ and refer to them as MRWs as fractional Wiener integrals or simply MRWs if there is no confusion. The case of $\mathrm{fBm}$ is much more complex than that of Bm because fractional Wiener integrals (integrals of deterministic functions with respect to $\mathrm{fBm}$, as in (1.2) conditional on $Q$ ) are more involved than usual Wiener integrals. These integrals, especially when integrands are random, have been studied in depth only recently (see Carmona, Coutin and Montseny (2003), Decreusfond and Üstünel (1999), Gripenberg and Norros (1996), Pipiras and Taqqu (2000)). We suppose that $Q$ in (1.2) is an infinitely divisible cascading (IDC) noise or simply an infinitely divisible cascade as introduced in Muzy and Bacry (2002), Chainais et al. $(2005 a)$. This appears to be the most general class of stationary multifractal noise processes up to date.

When working with fractional integrals, it is convenient to use a different parametrization for fBm. We set

$$
\kappa=H-\frac{1}{2}
$$


and write $B^{\kappa}=B_{H}$. Observe that the values $0,(-1 / 2,0)$ and $(0,1 / 2)$ of $\kappa$ are now associated with the values $1 / 2,(0,1 / 2)$ and $(1 / 2,1)$ of $H$.

The IDC noise $Q$ is viewed as a limit of a family of processes $Q_{r}$ when $r \rightarrow 0$ and is characterized by a function $\varphi(q)$ (cf. Section 2.1 for more details). MRW is defined as fractional Wiener integral with $r \rightarrow 0$ (cf. Eq. (2.33)).

MRWs as fractional Wiener integrals are first discussed in passing by Muzy and Bacry (2002), Bacry, Delour and Muzy (2001). More recently, they are studied in greater depth by Ludeña (2008) where they are called Multifractal Fractional Random Walks. Despite some overlap, the focus of this work is quite different from Ludeña (2008). First, we take a closer look at the definition of MRWs. These objects were defined in previous works when

$$
2 \kappa+c \varphi(2)>0 \quad \Leftrightarrow \quad \kappa>-\frac{c \varphi(2)}{2}=: \kappa_{c},
$$

where $\varphi(q)$ is the function mentioned above and $c$ is a constant entering the construction of an IDC noise. (Since $\varphi(2)<0,(1.5)$ implicitly assumes that $\kappa>0$ and $\kappa_{c}<1 / 2$.) We study here also the case when (1.5) does not hold, that is,

$$
\kappa \leq \kappa_{c},
$$

for both $\kappa>0$ and $\kappa<0$. We suggest that, in case (1.6), a suitable normalization should be used for (1.2) with $Q_{r}$ replacing $Q$ as $r \rightarrow 0$. This is supported by proving convergence of moments for the expected range of parameters, and by empirical evidence in simulations. Somewhat surprisingly, the inequality (1.6) is even simpler to deal with in moment calculations, and scaling properties of MRWs for $\kappa \leq \kappa_{c}$ essentially correspond to those of MRWs defined through the usual Brownian motion. Second, we provide natural conditions on finiteness of (even order $2 p$ ) moments of MRWs, which were conjectured by Ludeña (2008), namely,

$$
\left(\kappa+\frac{1}{2}\right) 2 p-1+c \varphi(2 p)>0
$$

under the condition (1.5). This is achieved through a direct approach (covering both (1.5) and (1.6) for $\kappa>0$ ) whereas Ludeña (2008) used power counting methods leading to stronger conditions. Third, we illustrate a number of our results (such as convergence, scaling properties) through numerical simulations. In particular, this explores the practical relevance of some theoretical results that are asymptotic in nature, and raises other questions such as oversampling in simulation of MRWs. Giving a proper credit, it should be said, however, that Ludeña (2008) addresses an important question of asymptotic behavior of $p$-variations of MRWs. This is not the focus in this work.

The rest of the paper is organized as follows. The next section, Section 2, contains some preliminaries on infinitely divisible cascades and fractional Wiener integrals. Definitions of MRWs are studied in Section 3. Properties of MRWs can be found in Section 4. The proofs of several auxiliary results are moved to Section 5 .

\section{Preliminaries}

\subsection{Infinitely divisible cascading noise}

We recall here the definition of Infinitely Divisible Cascading (IDC) noise from Chainais et al. (2005a) that will be used for integrands $Q$ in the definition of (1.2).

Infinitely divisible measure. Let $M$ be an infinitely divisible, independently scattered random measure on

$$
\mathbb{P}^{+}=\mathbb{R} \times \mathbb{R}^{+}=\{(t, r): t \in \mathbb{R}, r>0\}
$$


with the generating infinitely divisible distribution $G$ having a moment generating function

$$
\int_{\mathbb{R}} e^{q x} G(d x)=e^{-\rho(q)}
$$

and the control measure

$$
d m(t, r)=d t \mu(d r)
$$

on $\mathbb{P}^{+}$. Independent scatteredness means that random variables $M\left(A_{1}\right), \ldots, M\left(A_{n}\right), n \geq 1$, are independent whenever Borel sets $A_{i} \subset \mathbb{P}^{+}$are mutually disjoint. Infinite divisibility with the generating function $G$ and the control measure $m$ means that the moment generating function of $M(A)$ with Borel set $A \subset \mathbb{P}^{+}$is given by

$$
E e^{q M(A)}=e^{-\rho(q) m(A)} .
$$

Infinitely divisible noise. By definition, an IDC noise is a family of processes $Q_{r}(t), r>0, t \in \mathbb{R}$, defined by

$$
Q_{r}(t)=\frac{e^{M\left(C_{r}(t)\right)}}{E e^{M\left(C_{r}(t)\right)}},
$$

where

$$
C_{r}(t)=\left\{\left(t^{\prime}, r^{\prime}\right): r \leq r^{\prime} \leq 1, t-\frac{r^{\prime}}{2} \leq t^{\prime}<t+\frac{r^{\prime}}{2}\right\}
$$

is a cone in $\mathbb{P}^{+}$. For each $r>0$, the process $Q_{r}(t), t \in \mathbb{R}$, is stationary. We also let

$$
Q_{r}^{s}(u)=\frac{Q_{r}(u)}{Q_{s}(u)}
$$

which will be used in the proof of one of the main results, and set

$$
\varphi(q)=\rho(q)-q \rho(1) .
$$

Note that $\varphi(0)=\varphi(1)=0$ and $\varphi$ is concave. Moreover, $\varphi(2)<0$. The trivial case $\rho(q)=0$ (yielding $\left.Q_{r}(t) \equiv 1\right)$ is excluded throughout.

Examples of underlying distributions $G$ are Gaussian, stable, gamma, compound Poisson laws. Several useful formulas regarding IDC noise are

$$
\begin{gathered}
E Q_{r}(t)^{q}=e^{-\varphi(q) m_{r}(0)}, \\
E Q_{r}(t) Q_{r}(s)=e^{-\varphi(2) m_{r}(|t-s|)},
\end{gathered}
$$

where

$$
m_{r}(u)=m\left(C_{r}(0) \cap C_{r}(u)\right), \quad u \geq 0, r>0 .
$$

(Relation (2.10) implicitly assumes that $\varphi(2)$ exists. This may not be the case for some underlying distributions $G$ such as most of stable distributions.) More generally, as in Lemma 1 of Bacry and Muzy (2003), p. 457, one has, for $0 \leq z_{1} \leq z_{2} \leq \ldots \leq z_{n}$,

$$
E Q_{r}\left(z_{1}\right) Q_{r}\left(z_{2}\right) \ldots Q_{r}\left(z_{n}\right)=\exp \left\{-\sum_{1 \leq i<j \leq n} \alpha_{j-i+1} m_{r}\left(z_{j}-z_{i}\right)\right\}
$$

where

$$
\alpha_{k}=\varphi(k)-2 \varphi(k-1)+\varphi(k-2), \quad k \geq 2 .
$$

Since $\varphi$ is a concave function, one has $\alpha_{k} \leq 0$. 
Scale invariance and infinitely divisible noise. Throughout this work, we consider only those infinitely divisible noises whose control measure $m$ is given by either

$$
d m(t, r)=\left\{\begin{array}{cl}
d t \frac{c d r}{r^{2}}, & \text { if } 0<r \leq 1 \\
0, & \text { if } r>1
\end{array}\right.
$$

or

$$
d m(t, r)=\left\{\begin{array}{cl}
d t\left(\frac{c d r}{r^{2}}+c \delta_{\{1\}}(d r)\right), & \text { if } 0<r \leq 1, \\
0, & \text { if } r>1,
\end{array}\right.
$$

where $c>0$ is a constant and $\delta_{\{1\}}(d r)$ denotes a point mass at $r=1$. With this choice, we follow Muzy and Bacry $(2002,2003)$, Chainais et al. $(2005 a, 2005 b)$. The measures (2.14)-(2.15) are such that $m_{r}(0)$ behaves as $-c \ln r$ when $r \rightarrow 0$ (cf. (5.1))-(5.2)), and are expected to lead to processes with truly multifractal properties. The cases (2.14)-(2.15) are known as those of scale invariance. The case (2.15), considered in Bacry and Muzy $(2002,2003)$, is also referred to as that of exact scale invariance.

In the case of exact scale invariance, in particular, for $t \in(0,1)$,

$$
\left\{Q_{r t}(t u)\right\}_{u \in \mathbb{R}} \stackrel{d}{=} e^{\Omega_{t}}\left\{Q_{r}(u)\right\}_{u \in \mathbb{R}},
$$

where $=_{d}$ stands for the equality of finite dimensional distributions and $\Omega_{t}$ is a random variable which is independent of $\left\{Q_{r}(u)\right\}_{u \in \mathbb{R}}$ and has the moment generating function

$$
E e^{q \Omega_{t}}=t^{c \varphi(q)},
$$

where $\varphi(q)$ is given by (2.8). In the scale invariant case (2.14), using the notation (2.7), for $0<r<$ $b<1$,

$$
\left\{Q_{r}^{b}(b u)\right\}_{u \in \mathbb{R}} \stackrel{d}{=}\left\{Q_{r / b}(u)\right\}_{u \in \mathbb{R}} .
$$

Again, only the cases (2.14)-(2.15) are considered in this work, and we will generally make no further reference to this, unless the focus is specifically on (2.14) or (2.15).

Multifractal infinitely divisible motion. A truly multifractal process, called IDC motion in Chainais et al. (2005a), p. 1068, is obtained by integrating $Q_{r}(t)$ over $t$ and letting $r \rightarrow 0$, namely,

$$
X(t)=\lim _{r \rightarrow 0} \int_{0}^{t} Q_{r}(u) d u .
$$

We shall substitute $Q_{r}$ into the integral (1.2) for $Q$ and also let $r \rightarrow 0$. Note that the limit is outside the integral sign in (2.19). In fact, the limiting object $\lim _{r \rightarrow 0} Q_{r}(u)$ is trivial: it converges to 0 almost surely and for almost all $u$ (see Section III.A of Chainais et al. (2005a)). The limiting integral (2.19), on the other hand, is non-degenerate under suitable (and mild) assumptions. In case of scale invariant cascades, the corresponding assumption is

$$
\exists \epsilon>1: \zeta_{\mathrm{IDC}}(1+\epsilon)>1,
$$

where

$$
\zeta_{\mathrm{IDC}}(q)=q+c \varphi(q)
$$

or since $\zeta_{\mathrm{IDC}}(1)=1$ and assuming $\zeta_{\mathrm{IDC}}$ is smooth,

$$
\zeta_{\text {IDC }}^{\prime}(1)=1+c \varphi^{\prime}(1)>0
$$

(see Barral and Mandelbrot (2002), Bacry and Muzy (2003)). 


\section{$2.2 \quad$ Fractional Wiener integrals}

We recall here several known facts on fractional Wiener integrals $\int_{0}^{t} f(u) d B^{\kappa}(u)$ that will be used in the paper, where $B^{\kappa}, \kappa \in(-1 / 2,1 / 2)$, is fractional Brownian motion (fBm), $f$ is a deterministic function and $t \in \mathbb{R}$ is fixed. Most of the facts below are taken from a review article by Pipiras and Taqqu (2003). We also exclude below the case $\kappa=0$ for which fractional Wiener integral is well-known to be defined when $f \in L^{2}[0, t]$.

When $\kappa \in(0,1 / 2)$, several classes of integrands $f$ are available for fractional Wiener integrals. One such class is defined as

$$
\mathcal{L}^{\kappa}=\left\{f: \int_{0}^{t} \int_{0}^{t}|f(u)||f(v)||u-v|^{2 \kappa-1} d u d v<\infty\right\}
$$

It can be shown to be an inner product space with the inner product

$$
\langle f, g\rangle_{\mathcal{L}^{\kappa}}=C_{\kappa} \int_{0}^{t} \int_{0}^{t} f(u) g(v)|u-v|^{2 \kappa-1} d u d v
$$

where $C_{\kappa}>0$ is a constant. (The inner product space is known to be incomplete.)

When $\kappa \in(-1 / 2,0)$, the commonly used class of integrands $f$ is defined as

$$
\mathcal{L}^{\kappa}=\left\{f: \exists \phi_{f} \in L^{2}[0, t]: f(u)=u^{-\kappa}\left(I_{t-}^{-\kappa} s^{\kappa} \phi_{f}(s)\right)(u)\right\} .
$$

It can be shown to be a Hilbert space with the inner product

$$
\begin{aligned}
\langle f, g\rangle_{\mathcal{L}^{\kappa}} & =C_{\kappa} \int_{0}^{t} s^{-2 \kappa}\left(I_{t-}^{\kappa} u^{\kappa} f(u)\right)(s)\left(I_{t-}^{\kappa} u^{\kappa} g(u)\right)(s) d s \\
& =C_{\kappa} \int_{0}^{t} \phi_{f}(s) \phi_{g}(s) d s,
\end{aligned}
$$

where, for $\alpha>0$,

$$
\left(I_{t-}^{\alpha} g\right)(s)=\frac{1}{\Gamma(\alpha)} \int_{0}^{t} g(u)(u-s)_{+}^{\alpha-1} d u, \quad s \in[0, t],
$$

is a fractional integral of order $\alpha$ and, for $\alpha \in(-1,0)$,

$$
\left(I_{t-}^{\alpha} g\right)(u)=-\frac{1}{\Gamma(\alpha+1)} \frac{d}{d u} \int_{0}^{t} g(s)(s-u)_{+}^{\alpha} d s, \quad u \in[0, t],
$$

is a fractional derivative of order $(-\alpha)$ (or fractional integral of negative order $\alpha$ ).

For $f \in \mathcal{L}^{\kappa}$, a fractional Wiener integral, denoted as

$$
\mathcal{I}^{\kappa}(f)=\int_{0}^{t} f(u) d B^{\kappa}(u), \quad t \in \mathbb{R}
$$

is defined in the usual way as the $L^{2}(\Omega)$-limit of integrals $\mathcal{I}^{\kappa}\left(f_{n}\right)$ for elementary functions $f_{n}$ such that $\left\langle f-f_{n}, f-f_{n}\right\rangle_{\mathcal{L}^{\kappa}} \rightarrow 0$. (An elementary function $g$ has a form $g(u)=\sum_{k=1}^{n} g_{k} 1_{\left[a_{k}, b_{k}\right)}(u)$ and its fractional Wiener integral is defined as $\mathcal{I}^{\kappa}(g)=\sum_{k=1}^{n} g_{k}\left(B^{\kappa}\left(b_{k}\right)-B^{\kappa}\left(a_{k}\right)\right)$.) By definition, $\mathcal{I}^{\kappa}(f)$, $f \in \mathcal{L}^{\kappa}$, are Gaussian random variables with the covariance structure

$$
E \mathcal{I}^{\kappa}(f) \mathcal{I}^{\kappa}(g)=\langle f, g\rangle_{\mathcal{L}^{\kappa}}
$$

Fractional derivatives and hence the case $\kappa \in(-1 / 2,0)$ are generally more difficult to deal with. The following formula, however, often facilitates computations even in the case $\kappa \in(-1 / 2,0)$. For "nice" functions $g$ and $\alpha \in(-1,0)$,

$$
\left(I_{t-}^{\alpha} g\right)(u)=\frac{g(u)(t-u)^{\alpha}}{\Gamma(\alpha+1)}+\frac{1}{\Gamma(\alpha)} \int_{0}^{t}(g(s)-g(u))(s-u)_{+}^{\alpha-1} d s .
$$


"Nice" means here, for example, step or $C^{1}[0, t]$ functions. Observe that, unlike (2.29), the relation (2.32) does not involve derivatives. Another instance where the relation (2.32) holds is the following.

Lemma 2.1 Let $\alpha \in(-1 / 2,0)$ and $g \in L^{2}[0, t]$ be such that $g_{\|}^{(\alpha)} \in L^{2}[0, t]$, where

$$
g_{\|}^{(\alpha)}(u)=\frac{|g(u)|(t-u)^{\alpha}}{\Gamma(\alpha+1)}+\frac{1}{\Gamma(\alpha)} \int_{0}^{t}|g(s)-g(u)|(s-u)_{+}^{\alpha-1} d s .
$$

Then, $I_{t-}^{\alpha} g$ is well defined, given by (2.32) and belongs to $L^{2}[0, t]$.

Proof: For $\varepsilon \in[0, t]$, consider

$$
g_{\varepsilon}^{(\alpha)}(u)=g(u) \frac{(t+\varepsilon-u)^{\alpha}}{\Gamma(\alpha+1)}+\frac{1}{\Gamma(\alpha)} \int_{u}^{t}(g(s)-g(u))(s-u+\varepsilon)^{\alpha-1} d s .
$$

Then $g_{\varepsilon}^{(\alpha)}$ belongs to $L^{2}[0, t]$ and converges to $g_{0}^{(\alpha)}$ in $L^{2}[0, t]$. Moreover,

$$
g_{\varepsilon}^{(\alpha)}(u)=\frac{\varepsilon^{\alpha} g(u)}{\Gamma(\alpha+1)}+\frac{1}{\Gamma(\alpha)} \int_{u}^{t} g(s)(s+\varepsilon-u)^{\alpha-1} d s .
$$

These observations and the Fubini's theorem (for the second equality below) allow us to conclude that

$$
\begin{aligned}
\int_{v}^{t} g_{0}^{(\alpha)}(u) d u & =\lim _{\varepsilon \rightarrow 0} \int_{v}^{t} g_{\varepsilon}^{(\alpha)}(u) d u \\
& =\lim _{\varepsilon \rightarrow 0} \frac{1}{\Gamma(\alpha+1)} \int_{v}^{t} g(s)(s-v+\varepsilon)^{\alpha} d s \\
& =\frac{1}{\Gamma(\alpha+1)} \int_{v}^{t} g(s)(s-v)^{\alpha} d s .
\end{aligned}
$$

This implies that $I_{t-}^{\alpha} g$ is well defined and equals $g_{0}^{(\alpha)}$.

Corollary 2.1 For $\kappa \in(-1 / 2,0)$, if $g \in L^{2}[0, t]$ and if $g_{\|}^{[\kappa]} \in L^{2}[0, t]$, where

$$
g_{\|}^{[\kappa]}(u)=\frac{|g(u)|(t-u)^{\kappa}}{\Gamma(\kappa+1)}+\frac{u^{-\kappa}}{\Gamma(\kappa)} \int_{0}^{t}\left|s^{\kappa} g(s)-u^{\kappa} g(u)\right|(u-s)_{+}^{\kappa-1} d s,
$$

then $g$ belongs to $\mathcal{L}^{\kappa}$ and

$$
\phi_{g}(u)=\frac{g(u)(t-u)^{\kappa}}{\Gamma(\kappa+1)}+\frac{u^{-\kappa}}{\Gamma(\kappa)} \int_{0}^{t}\left(s^{\kappa} g(s)-u^{\kappa} g(u)\right)(u-s)_{+}^{\kappa-1} d s .
$$

\subsection{Fractional Wiener integrals of IDC noise}

In Section 3, we shall consider MRWs as the limits of fractional Wiener integrals

$$
Z_{r}^{\kappa}(t)=\int_{0}^{t} Q_{r}(u) d B^{\kappa}(u)
$$

as $r \rightarrow 0$, where $Q_{r}(u)$ is an IDC noise defined in Section 2.1 and $B^{\kappa}$ is $\mathrm{fBm}$. Using the preliminaries of Sections 2.1 and 2.2, we state here that (2.33) is well-defined for each fixed $r>0$. See Section 5 for a proof.

Proposition 2.1 For $\kappa \in(-1 / 2,1 / 2), Q_{r} \in \mathcal{L}^{\kappa}$ a.s. for each fixed $r>0$ and hence fractional Wiener integral (2.33) is well-defined a.s. for each $r>0$. 


\subsection{Important notation and cases}

Throughout this work, we consider only the case of scale invariance (2.14) or (2.15). It is convenient to introduce the following notation and cases. Let

$$
\kappa_{c}=-c \varphi(2) / 2>0 .
$$

Consider the cases

$$
\begin{aligned}
& (A): c \varphi(2)+2 \kappa>0 \quad \Leftrightarrow \quad \kappa_{c}<\kappa<1 / 2, \\
& (E): c \varphi(2)+2 \kappa=0 \quad \Leftrightarrow \quad \kappa=\kappa_{c}, \\
& (B): c \varphi(2)+2 \kappa<0, \kappa \geq 0 \quad \Leftrightarrow \quad 0 \leq \kappa<\kappa_{c}, \\
& (N): c \varphi(2)+2 \kappa<0, \kappa<0 \quad \Leftrightarrow \quad-1 / 2<\kappa<0,
\end{aligned}
$$

where the notation $(A),(E)$ and $(B)$ stand for 'Above', 'Equal to' and 'Below' the critical value $\kappa_{c}$, respectively, while $(N)$ indicates 'Negative' for $\kappa<0$. Let also

$$
\begin{aligned}
& \zeta(q)=\left\{\begin{array}{clc}
\left(\kappa+\frac{1}{2}\right) q+c \varphi(q), & & \text { when }(A), \\
(1-c \varphi(2)) \frac{q}{2}+c \varphi(q)=\left(\kappa_{c}+\frac{1}{2}\right) q+c \varphi(q), & & \text { when }(E) \text { or }(B) \text { or }(N),
\end{array}\right. \\
& =\left(\kappa^{*}+\frac{1}{2}\right) q+c \varphi(q) \text {, }
\end{aligned}
$$

where

$$
\kappa^{*}=\max \left\{\kappa, \kappa_{c}\right\} .
$$

The function $\zeta(q)$ will play the role of scaling exponents. The cases $(E)$ and $(B)$ will be refined below.

\section{MRW: definition, convergence and numerical synthesis}

We wish to define Multifractal Random Walk (MRW) as the limit of fractional integrals $Z_{r}^{\kappa}(t)$ in (2.33), as $r \rightarrow 0$. The following section, Section 3.1, contains some preliminary technical results. Various types of convergence are considered in Section 3.2, and numerical synthesis is discussed in Section 3.3. Most of stated results of this section are proved in Section 5.

\subsection{Calculations of even order moments}

The following technical result concerns even order moments of $Z_{r}^{\kappa}(t)$ when $\kappa \in(0,1 / 2)$.

Proposition 3.1 Suppose $p \in \mathbb{N}$ is such that

$$
\zeta(2 p)>1,
$$

where $\zeta$ is defined in (2.39). Then, for fixed $t \in \mathbb{R}$, as $r \rightarrow 0$,

$$
E\left|Z_{r}^{\kappa}(t)\right|^{2 p} \sim C(p, t) n(r)^{2 p}
$$

where

$$
n(r)^{2}=\left\{\begin{array}{cl}
1, & \text { when }(A), \\
\ln r^{-1}, & \text { when }(E), \\
r^{c \varphi(2)+2 \kappa}, & \text { when }(B)
\end{array}\right.
$$

and $C(p, t)$ is a non-zero constant which depends on $p, t$ and also on $\kappa, c$ and $\varphi$. 
Note that $n(r)$ in (3.3) diverges as $r \rightarrow 0$ in cases $(E)$ and $(B)$. The case $\kappa \in(-1 / 2,0)$ is much more cumbersome, and only the case of $p=1$ (second moment) is considered.

Proposition 3.2 In case $(N)$, for fixed $t \in \mathbb{R}$, as $r \rightarrow 0$,

$$
E\left|Z_{r}^{\kappa}(t)\right|^{2} \sim C(t) n(r)^{2}:=C(t) r^{c \varphi(2)+2 \kappa},
$$

where $C(t)$ is a non-zero constant which depends on $t$ and also on $\kappa, c$ and $\varphi(2)$.

The relations (3.2) and (3.4) above suggest that $Z_{r}^{\kappa}$ may need to be normalized for convergence when $0 \leq \kappa \leq \kappa_{c}$ or $\kappa<0$. Hence, let also

$$
\widetilde{Z}_{r}^{\kappa}(t)=\frac{Z_{r}^{\kappa}(t)}{n(r)}
$$

where $n(r)$ is defined in (3.3) and (3.4).

\subsection{Various types of convergence}

We discuss here several types of convergence of the process $\widetilde{Z}_{r}^{\kappa}(t)$ defined in (3.5).

\subsubsection{Convergence of moments}

Possibly the weakest type of convergence is that of (absolute) moments. Let $\mathcal{M}$ be a subset of $\mathbb{R}$. We say that the $\mathcal{M}$ moments of $X_{r}$ converge to those of $X$ (the $\mathcal{M}$ moments of $X_{r}$ converge, resp.), if $E\left|X_{r}\right|^{m} \rightarrow E|X|^{m}$ for $m \in \mathcal{M}\left(E\left|X_{r}\right|^{m}\right.$ converge for $m \in \mathcal{M}$, resp.). Though the moment convergence is weak, it is quite appropriate for multifractals where their scaling properties are defined in terms of absolute moments.

Proposition 3.1 can now be restated as follows, and requires no separate proof.

Theorem 3.1 In cases $(A),(E)$ and $(B)$, the $\mathcal{M}$ moments of $\widetilde{Z}_{r}^{\kappa}(t)$ converge, where

$$
\mathcal{M}=\{2 p: p \in \mathbb{N}, \zeta(2 p)>1\}
$$

and $\zeta$ is defined by (2.39).

Remark 3.1 The convergence of moments in Theorem 3.1 can be extended to that of linear combinations of finite dimensional distributions of $\widetilde{Z}_{r}^{\kappa}$, that is, $a_{1} \widetilde{Z}_{r}^{\kappa}\left(t_{1}\right)+\ldots+a_{n} \widetilde{Z}_{r}^{\kappa}\left(t_{n}\right), a_{i}, t_{i} \in \mathbb{R}$, $i=1, \ldots, n$.

In case $(N)$, by Proposition 3.2 , the second moment of $\widetilde{Z}_{r}^{\kappa}(t)$ converges by construction. We conjecture that all $\mathcal{M}$ moments specified in Theorem 3.1 also converge.

\subsubsection{Convergence in $\mathrm{L}^{2}$}

Another useful type of convergence is that in $L^{2}(\Omega)$. In this regard, the following result is useful.

Lemma 3.1 For $r \geq r^{*}>0$, we have $E Z_{r}^{\kappa}(t) Z_{r^{*}}^{\kappa}(t)=E Z_{r}^{\kappa}(t)^{2}$, where $Z_{r}^{\kappa}(t)$ is defined by (2.33).

Propositions 3.1 and 3.2, and Lemma 3.1 above lead to the following result.

Theorem 3.2 For fixed $t \in \mathbb{R}, \widetilde{Z}_{r}^{\kappa}(t)$ converges in $L^{2}(\Omega)$ in case $(A)$, and does not converge in $L^{2}(\Omega)$ in cases $(E),(B)$ and $(N)$. 
Proof: In case $(A)$, we have $\widetilde{Z}_{r}^{\kappa}(t)=Z_{r}^{\kappa}(t)$. Observe by Lemma 3.1 that, for $r \geq r^{*}>0$,

$$
E\left(Z_{r}^{\kappa}(t)-Z_{r^{*}}^{\kappa}(t)\right)^{2}=E Z_{r^{*}}^{\kappa}(t)^{2}-E Z_{r}^{\kappa}(t)^{2} .
$$

Hence, there is convergence in $L^{2}(\Omega)$ if and only if $E Z_{r}^{\kappa}(t)^{2}$ converges. This happens under $(A)$ by Proposition 3.1.

For the last statement of the theorem, consider, for example, the case $(E)$ with $n(r)=\sqrt{\ln r^{-1}}$. Then, by Lemma 3.1, for $r \geq r^{*}$,

$$
E\left(\widetilde{Z}_{r}^{\kappa}(t)-\widetilde{Z}_{r^{*}}^{\kappa}(t)\right)^{2}=E\left(\frac{Z_{r}^{\kappa}(t)}{\sqrt{\ln r^{-1}}}-\frac{Z_{r^{*}}^{\kappa}(t)}{\sqrt{\ln r^{*-1}}}\right)^{2}=\frac{E Z_{r}^{\kappa}(t)^{2}}{\ln r}\left(2 \sqrt{\frac{\ln r}{\ln r^{*}}}-1\right)-\frac{E Z_{r^{*}}^{\kappa}(t)^{2}}{\ln r^{*}} .
$$

By Proposition 3.1, $E Z_{r}^{\kappa}(t)^{2} / \ln r, E Z_{r^{*}}^{\kappa}(t)^{2} / \ln r^{*}$ converge to the same non-zero constant as $r, r^{*} \rightarrow$ 0 . But $r^{*}, r$ can be taken such that $\lim \frac{\ln r}{\ln r^{*}}=a<1$, showing that $\widetilde{Z}_{r}^{\kappa}(t)$ does not converge in $L^{2}(\Omega)$.

Remark 3.2 In case $(A)$ when there is convergence in $L^{2}(\Omega)$, one may be inclined to write and to define the limit MRW as $\int_{0}^{t} Q_{0}(u) d B^{\kappa}(u)$. This is impossible because $Q_{0}(u)$ is not defined as a regular process. (See also the discussion following (2.19).) On another hand, one could still expect that the $\operatorname{limit}_{r} \lim _{0}^{t} Q_{r}(u) d B^{\kappa}(u)$ is characterized by the IDC motion $X$ defined in (2.19). In fact, Ludeña (2008) defines the limit as being conditionally Gaussian with the covariance structure

$$
\int_{0}^{t} \int_{0}^{s}|u-v|^{2 \kappa-1} X(d u) X(d v)
$$

The appearance of this covariance structure suggests that the above integral is well-defined (a.s.) for IDC motion $X$. Since $|u-v|^{2 \kappa-1}$ blows up at $u=v$, this obviously imposes smoothness conditions on the motion $X$. A closer look at the argument of Ludeña (2008) shows that (3.9) is understood as the limit of $\int_{0}^{t} \int_{0}^{s} Q_{r}(u) Q_{r}(v)|u-v|^{2 \kappa-1} d u d v$ as $r \rightarrow 0$. Whether it can be interpreted through $X$ itself is an open question.

Remark 3.3 Observe that convergence in $L^{2}(\Omega)$ for MRWs (case $\left.(A)\right)$ implies the condition (2.22) for non-degeneracy of IDC motion. Indeed, in case $(A)$, one has $\zeta(1)=\kappa+1 / 2<1, \zeta(2)=$ $2\left(\kappa-\kappa_{c}\right)+1>1$ and concave $\zeta(q)$. Assuming smoothness of $\zeta$, the latter conditions can happen only when $\zeta^{\prime}(1)>0$. But $\zeta^{\prime}(1)=\kappa+1 / 2+c \varphi^{\prime}(1)<1+c \varphi^{\prime}(1)=\zeta_{\text {IDC }}^{\prime}(1)$ and hence $\zeta_{\text {IDC }}^{\prime}(1)>0$, that is, $(2.22)$ holds. We do not believe this argument could be reversed in general.

Remark 3.4 $L^{2}(\Omega)$-convergence and non-convergence in Theorem 3.2 can also be viewed from the following interesting angle. For example, when $\kappa=0$, if $\int_{0}^{t} r^{-c \varphi(2) / 2} Q_{r}(u) d B^{0}(u)$ converged, one would have that

$$
\begin{gathered}
E\left(\int_{0}^{t} r_{1}^{-c \varphi(2) / 2} Q_{r_{1}}(u) d B^{0}(u)-\int_{0}^{t} r_{2}^{-c \varphi(2) / 2} Q_{r_{2}}(u) d B^{0}(u)\right)^{2} \\
=E \int_{0}^{t}\left(r_{1}^{-c \varphi(2) / 2} Q_{r_{1}}(u)-r_{2}^{-c \varphi(2) / 2} Q_{r_{2}}(u)\right)^{2} d u \rightarrow 0
\end{gathered}
$$

as $r_{1}, r_{2} \rightarrow 0$. Completeness of $L^{2}(\Omega \times[0, t])$ would imply that $r^{-c \varphi(2) / 2} Q_{r}(u)$ converges to a regular process in $L^{2}(\Omega \times[0, t])$. But this would not be possible since $r^{-c \varphi(2) / 2} Q_{r}(u)$ is expected highly irregular as $r \rightarrow 0$. The same argument could also be applied when $\kappa<0$ for which the integrand space of $\mathrm{fBm}$ is complete but not for $\kappa>0$ when there is no completeness (see Section 2.2). These arguments suggest that non-convergence in Theorem 3.2 is, in fact, expected when $\kappa \leq 0$, and that convergence in $L^{2}(\Omega)$ can only happen when $\kappa>0$. 

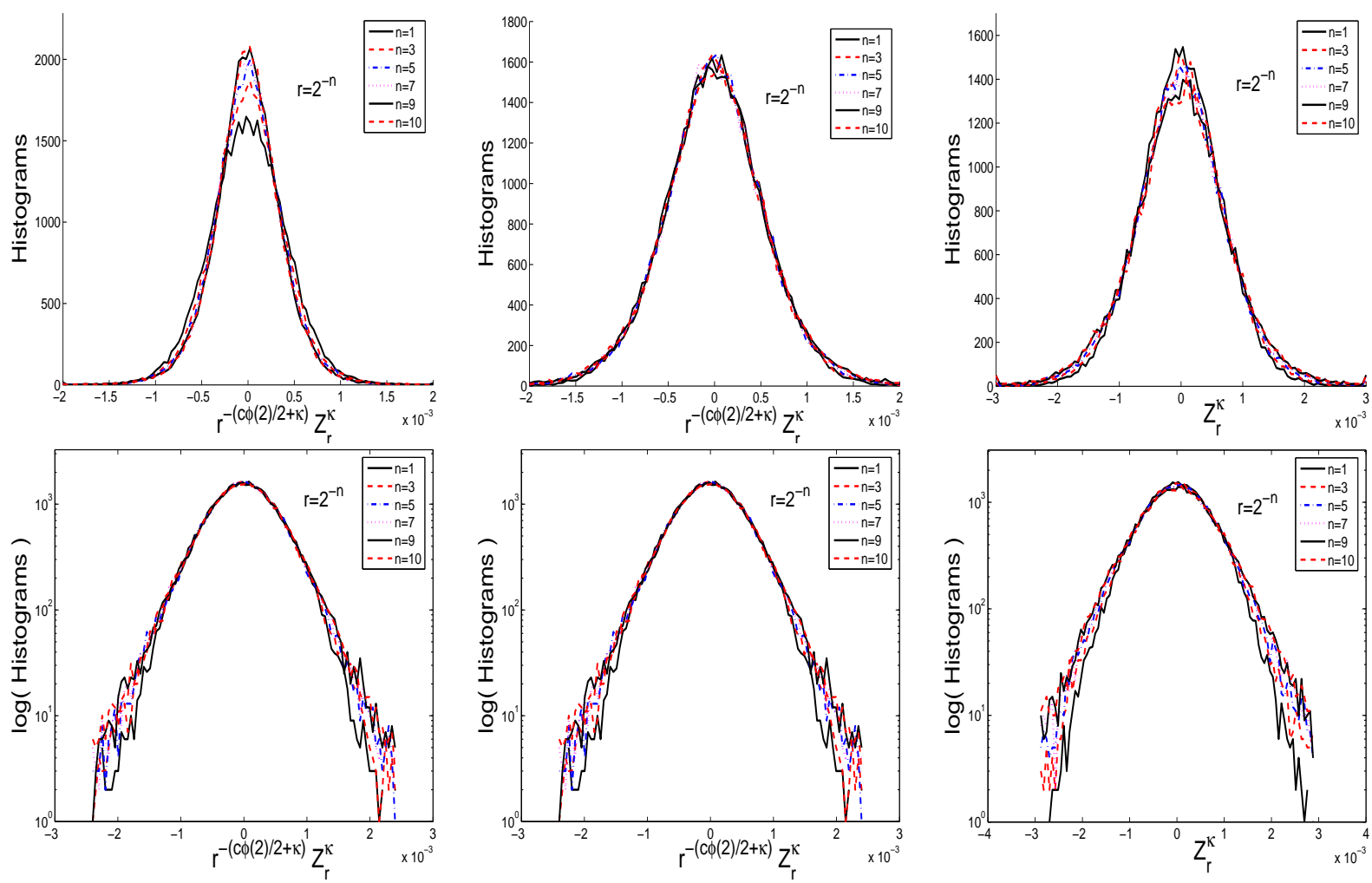

Figure 1: Histograms of $\tilde{Z}_{r}^{\kappa}(1)$ for various resolutions $r$ ranging from $1 / 1024$ to $1 / 2$. First row: histograms for (a) $\kappa=-0.1<0<\kappa_{c}$, (b) $0<\kappa=0.05<\kappa_{c}$, (c) $\kappa_{c}<\kappa=0.2$. Second row: log-histograms for (d) $\kappa=-0.1<0<\kappa_{c}$, (e) $0<\kappa=0.05<\kappa_{c}$, (f) $\kappa_{c}<\kappa=0.2$.

\subsubsection{Convergence in distribution}

Another type of convergence in a weaker sense is that in distribution. In fact, convergence of $\widetilde{Z}_{r}^{\kappa}(t)$ in distribution takes place when $\kappa=0$. (When $\kappa=0$, there is no convergence in $L^{2}(\Omega)$.) To see this in the scale invariant case $(2.14)$, observe that

$$
\begin{array}{r}
\widetilde{Z}_{r}^{0}(t)=\frac{Z_{r}^{0}(t)}{r^{c \varphi(2) / 2}}=r^{-c \varphi(2) / 2} \int_{0}^{t} Q_{r}(u) d B(u)=r^{-c \varphi(2) / 2} \int_{0}^{t} \frac{e^{M\left(C_{r}(u)\right)}}{E e^{M\left(C_{r}(u)\right)}} d B(u) \\
=r^{-c \rho(2) / 2} \int_{0}^{t} e^{M\left(C_{r}(u)\right)} d B(u)=\int_{0}^{t}\left(\frac{e^{2 M\left(C_{r}(u)\right)}}{E e^{2 M\left(C_{r}(u)\right)}}\right)^{1 / 2} d B(u)=: \int_{0}^{t} \widetilde{Q}_{r}(u)^{1 / 2} d B(u),
\end{array}
$$

where we used the fact that $E e^{q M\left(C_{r}(u)\right)}=r^{c \rho(q)}$. Here, $\widetilde{Q}_{r}(u)$ is another IDC noise characterized by $\widetilde{\rho}(q)=\rho(2 q)$ and $\widetilde{\varphi}(q)=\varphi(2 q)-q \varphi(2)$. The convergence in distribution of (3.10) is shown in Bacry and Muzy (2003), p. 473. The convergence takes place also in distribution in the space of functions.

We conjecture that the convergence in distribution also takes place in cases $(E),(B)$ and $(N)$. This is supported by the convergence of moments discussed in Section 3.2.1, and by the following numerical simulations. See Section 3.3 below for more details on numerical synthesis.

Fig. 1 shows histograms of $\widetilde{Z}_{r}^{\kappa}(1)$ for $r$ ranging from $1 / 2$ to $1 / 1024$, respectively in the case $\kappa<0<\kappa_{c}$ (case $\left.(N)\right), 0<\kappa<\kappa_{c}$ (case $\left.(B)\right)$ and $0<\kappa_{c}<\kappa$ (case $\left.(A)\right)$. Each histogram results from 40000 realizations of $\widetilde{Z}_{r}^{\kappa}(1)$ for underlying compound Poisson cascades $Q_{r}(t)$ such that $\varphi(2)=-0.24$ and $c=1$. Therefore, the critical situation corresponds to the choice $\kappa_{c}=-c \varphi(2) / 2=0.12$. In all cases, the histograms obtained for various resolutions $r$ superimpose quite clearly, and support the conjecture of convergence in distribution. 


\subsubsection{Finer convergence results in case (A)}

In case $(A)$, finer convergence than that in $L^{2}(\Omega)$ can be established.

Theorem 3.3 Consider the case $(A)$. Then, as $r \rightarrow 0$, the sequence of processes $\left\{\widetilde{Z}_{r}^{\kappa}(t)\right\}_{t \in[0,1]}$ converges almost surely (and hence in distribution) in the space of continuous functions $C([0,1], \mathbb{R})$.

\subsubsection{Convergence in $\mathbf{L}^{q}$ and refinement of earlier cases}

Various types of convergence above can be complemented by that in $L^{q}(\Omega), 1<q \leq 2$. We suppose $\kappa \in(0,1 / 2)$ and consider only the case of exact scale invariance (2.15) for simplicity. For $\kappa \in(0,1 / 2)$, let

$$
\widetilde{\zeta}(q)=\left(\kappa+\frac{1}{2}\right) q+c \varphi(q)
$$

Note that $\zeta(q)$ in $(2.39)$ and the function $\widetilde{\zeta}(q)$ coincide in case $(A)$ and are different in cases $(E)$ and $(B)$.

Theorem 3.4 Consider the case of exact scale invariance (2.15), and $\kappa \in(0,1 / 2)$. Suppose there is $1<q \leq 2$ such that

$$
\widetilde{\zeta}(q)>1
$$

Then, for all $t \in \mathbb{R}$,

$$
\sup _{r \in(0,1]} E\left|Z_{r}^{\kappa}(t)\right|^{q}<\infty .
$$

In particular, for all $t \in \mathbb{R}, Z_{r}^{\kappa}(t)$ converges almost surely and in $L^{q}(\Omega)$ as $r \rightarrow 0$.

Here is where Theorem 3.4 fits in with previous results. In case $(A)$, by Theorem 3.2 , there is convergence in $L^{2}(\Omega)$ and hence that in $L^{q}(\Omega)$ for all $1<q<2$. Convergence almost surely is also proved in Theorem 3.3. Hence, in this case, Theorem 3.4 does not yield any new results. On the other hand, new results emerge in cases $(E)$ and $(B)$. Two cases should now be distinguished:

Case 1: $\widetilde{\zeta}^{\prime}(2)>0$,

Case 2: $\widetilde{\zeta}^{\prime}(2)<0$, where $\widetilde{\zeta}^{\prime}(q)$ indicates the derivative of $\widetilde{\zeta}(q)$, and $\widetilde{\zeta}(q)$ is defined in (3.11).

In cases $(E)$ and $(B), \widetilde{\zeta}(q)=(\kappa+1 / 2) q+c \varphi(q)$ is concave and such that $\widetilde{\zeta}(0)=0, \widetilde{\zeta}(1)=$ $\kappa+1 / 2<1$ and $\widetilde{\zeta}(2)=1+2\left(\kappa-\kappa_{c}\right) \leq 1$. Moreover, $\zeta(q)=\left(\kappa_{c}+1 / 2\right) q+c \varphi(q)$ is also concave and such that $\zeta(0)=0, \zeta(1)=\kappa_{c}+1 / 2, \zeta(2)=1$ and $\widetilde{\zeta}(q) \leq \zeta(q)$. In Case 1 , note that $\zeta^{\prime}(2) \geq \widetilde{\zeta}^{\prime}(2)>0$. Then, by concavity, one expects that $\zeta(2 p)>1$ even for some $p>1$, and that condition (3.12) does not hold for any $1<q \leq 2$. This corresponds to the situation considered in Proposition 3.1 and Theorem 3.1.

On the other hand, in Case 2, by concavity, one expects that condition (3.12) might hold for some $1<q<2$. (In particular, in case $(E)$, one expects this condition to hold for $q$ sufficiently close to 2.) In this case, by Theorem 3.4, there is convergence almost surely and in $L^{q}(\Omega)$ without any normalization. Note also that, if condition (3.12) holds for some $1<q<2$, one cannot expect that $\zeta(2 p)>1$ for $p>1$. Indeed, supposing $\widetilde{\zeta}(q)>1$ for some $1<q<2$ yields $\zeta(q)>1$. Using concavity of $\zeta(q)$ and $\zeta(2)=1$, one expects that $\zeta(2 p)<1$ for $p>1$.

In order to distinguish between above cases 1 and 2 , we shall use the following notation. Cases $(E)$ and $(B)$ will refer to the earlier cases $(E)$ and $(B)$ when $\widetilde{\zeta}^{\prime}(2)>0$. When $\widetilde{\zeta}^{\prime}(2)<0$, cases $(E)$ and $(B)$ will be denoted as

$$
\left(E^{*}\right) \text { and }\left(B^{*}\right) \text {, }
$$

respectively. In cases $\left(E^{*}\right)$ and $\left(B^{*}\right)$, we set $\widetilde{Z}_{r}^{\kappa}(t)=Z_{r}^{\kappa}(t)$. 


\begin{tabular}{|c|c|l|}
\hline \hline Case & Parameter range & $\widetilde{Z}_{r}^{\kappa}$ converges in \\
\hline \hline$(A)$ & $\kappa_{c}<\kappa<1 / 2$ & $L^{2}(\Omega) ;$ distribution; (suitable) moments; almost surely \\
\hline$(E),(B)$ & $0 \leq \kappa \leq \kappa_{c}, \widetilde{\zeta}^{\prime}(2)>0$ & $\begin{array}{l}\text { (suitable) moments; not } L^{2}(\Omega) ; \text { distribution when } \kappa=0 ; \\
\text { conjectured for distribution when } \kappa>0\end{array}$ \\
\hline$\left(E^{*}\right),\left(B^{*}\right)$ & $\begin{array}{c}0 \leq \kappa \leq \kappa_{c}, \widetilde{\zeta^{\prime}}(2)<0 \\
\widetilde{\zeta}(q)>1 \text { for some } q \in(1,2)\end{array}$ & $L^{q}(\Omega) ;$ almost surely \\
\hline$(N)$ & $-1 / 2<\kappa<0$ & not $L^{2}(\Omega) ;$ conjectured for distribution and moments \\
\hline
\end{tabular}

Table 1: Convergence of $\widetilde{Z}_{r}^{\kappa}$ in the scale invariant case.

¿From a practical perspective, cases $(E)$ and $(B)$ appear to be more relevant than cases $\left(E^{*}\right)$ and $\left(B^{*}\right)$. First, most known examples of IDC noise, in fact, fall into cases $(E)$ and $(B)$. Second, in cases $\left(E^{*}\right)$ and $\left(B^{*}\right)$, one expects the limit $\widetilde{Z}^{\kappa}(t)$ of $\widetilde{Z}_{r}^{\kappa}(t)$ to have infinite second moment. For these reasons, in simulations and Section 4 below, we shall focus on cases $(E)$ and $(B)$, rather than cases $\left(E^{*}\right)$ and $\left(B^{*}\right)$.

\subsubsection{Summary of convergence}

In Table 1, we summarize the convergence results for $\widetilde{Z}_{r}^{\kappa}$. We will refer to the limit by the following name.

Definition 3.1 The limit process $\widetilde{Z}^{\kappa}$ of $\widetilde{Z}_{r}^{\kappa}$ will be called Multifractal Random Walk (MRW) as fractional Wiener integral or simply MRW, whenever it exists.

\subsubsection{Using arbitrary powers of IDC noise}

It is interesting to compare above definitions of MRWs to those when considering integrals

$$
Z_{r}^{\kappa, \beta}(t)=\int_{0}^{t}\left(Q_{r}(u)\right)^{\beta} d B^{\kappa}(u)
$$

where the IDC noise $Q_{r}(u)$ has a power $\beta>0$. When $\beta=1 / 2$, integrals (3.14) and their limits appear, for example, in Bacry and Muzy (2003), Ludeña (2008). As already hinted in Section 3.2.3, the case of integrals (3.14) can be reduced to that of integrals (2.33) considered above. We state this as an elementary result next.

Proposition 3.3 For $\beta>0$, we have

$$
Z_{r}^{\kappa, \beta}(t)=\int_{0}^{t}\left(Q_{r}(u)\right)^{\beta} d B^{\kappa}(u)=C_{0} r^{c \varphi(\beta)} \int_{0}^{t} \bar{Q}_{r}(u) d B^{\kappa}(u)=: C_{0} r^{c \varphi(\beta)} \bar{Z}_{r}^{\kappa}(t),
$$

where $C_{0}=e^{-c \varphi(\beta)}$ and $C_{0}=1$ in the cases (2.14) and (2.15), respectively, $\bar{Q}_{r}$ is an IDC noise characterized by the same control measure $m$ and

$$
\bar{\varphi}(q)=\varphi(\beta q)-q \varphi(\beta) .
$$

Proof: Observe that

$$
\left(Q_{r}(u)\right)^{\beta}=\frac{e^{\beta M\left(C_{r}(u)\right)}}{\left(E e^{M\left(C_{r}(u)\right)}\right)^{\beta}}=\frac{E e^{\beta M\left(C_{r}(u)\right)}}{\left(E e^{M\left(C_{r}(u)\right)}\right)^{\beta}} \frac{e^{\beta M\left(C_{r}(u)\right)}}{E e^{\beta M\left(C_{r}(u)\right)}}=: C_{0} r^{c \varphi(\beta)} \frac{e^{\bar{M}\left(C_{r}(u)\right)}}{E e^{\bar{M}\left(C_{r}(u)\right)}}=: C_{0} r^{c \varphi(\beta)} \bar{Q}_{r}(u),
$$


where $\bar{M}(A)=\beta M(A)$ is an infinitely divisible measure characterized by

$$
E e^{q \bar{M}(A)}=E e^{\beta q M(A)}=e^{-\rho(\beta q) m(A)}
$$

and hence $\bar{\rho}(q)=\rho(\beta q)$ and $\bar{\varphi}(q)=\bar{\rho}(q)-q \bar{\rho}(1)=\rho(\beta q)-q \rho(\beta)=\varphi(\beta q)-q \varphi(\beta)$.

As a consequence of Proposition 3.3 and Theorem 3.2, for example,

$$
r^{-c \varphi(\beta)} Z_{r}^{\kappa, \beta}(t)
$$

converges in $L^{2}(\Omega)$ when

$$
\kappa>-\frac{c \bar{\varphi}(2)}{2}=-\frac{c(\varphi(2 \beta)-2 \varphi(\beta))}{2} .
$$

In case $\beta=1 / 2$, this happens when $\kappa>c \varphi(1 / 2)$. A discussion related to this section can also be found in Section 4.6 below.

\subsection{Numerical synthesis}

The process $Z_{r}^{\kappa}$ in (2.33) is synthesized numerically as follows. To produce $N$ samples $Z_{r}^{\kappa}(n / N)$, $n=0, \ldots, N-1$, at equally spaced times on the interval $[0,1)$, we generate $K=R N$ equally spaced samples $B^{\kappa}(k / K)$ and $Q_{r}(k / K), k=0, \ldots, K-1$, of fBm $B^{\kappa}$ and IDC $Q_{r}$. The integer constant $R$ is referred to as the oversampling factor. Continuous sums in (2.33) are then approximated using Riemann sums:

$$
Z_{r}^{\kappa}\left(\frac{n}{N}\right) \simeq \sum_{k=1}^{n R} Q_{r}\left(\frac{k}{K}\right)\left(B^{\kappa}\left(\frac{k}{K}\right)-B^{\kappa}\left(\frac{k-1}{K}\right)\right) .
$$

The samples $B^{\kappa}(k / K)$ of fBm are synthesized numerically using the so-called circulant matrix embedding method. This is not detailed here and the reader is referred, e.g., to Bardet et al. (2003) for a complete introduction.

The synthesis of IDC $Q_{r}$ first requires the choices of theoretical quantities: an infinitely divisible measure $M$ in (2.2) with its control measure (2.3); the triangle-shaped cone in (2.6). In practice, the resolution parameter $r$ in $Q_{r}$ needs to be chosen such that $r \geq 1 / K$. The samples $Q_{r}(k / K)$ are generated using the algorithms thoroughly described in Chainais et al. (2005a) and not recalled here.

Examples of sample paths of $Z_{r}^{\kappa}$ are produced using a specific type of IDC referred to as compound Poisson cascades (CPC). The same sample path of the CPC $Q_{r}$, shown in Fig. 2, is used to produce the sample paths of $\widetilde{Z}^{\kappa}$ for the three different cases, $\kappa<0<\kappa_{c}, 0<\kappa<\kappa_{c}$ and $0<\kappa_{c}<\kappa$, cf. Fig. 3. For illustration purposes, Fig. 4 compares the sample paths of MRW $Z^{\kappa}$ against those of the so-called $\mathrm{fBm}$ in multifractal time

$$
V^{\kappa}(t)=B^{\kappa}(X(t)),
$$

where $X(t)$ is the IDC motion in (2.19), obtained using the same sample paths of $B^{\kappa}(t)$ and $Q_{r}(t)$. For definitions of fBm in multifractal time, the reader is referred to Mandelbrot (1999), Riedi (2003). It is analyzed in Chainais et al. (2005a) and Bacry and Muzy (2002, 2003). Note also that, thanks to Riemann sums (3.17), the numerical synthesis of MRWs is much easier than that of $\mathrm{fBm}$ in multifractal time, which is based on resampling of $\mathrm{fBm}$.

\section{Properties of MRWs}

We examine here several properties of MRWs. Since no type of convergence was established in case $(N)$ in Section 3.2, this case will be excluded from theoretical results below. 


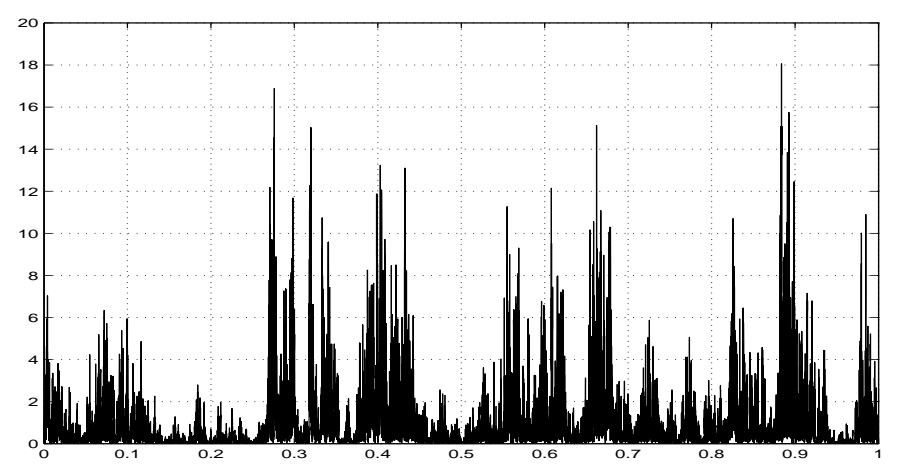

Figure 2: A sample path of IDC (CPC) $Q_{r}$.
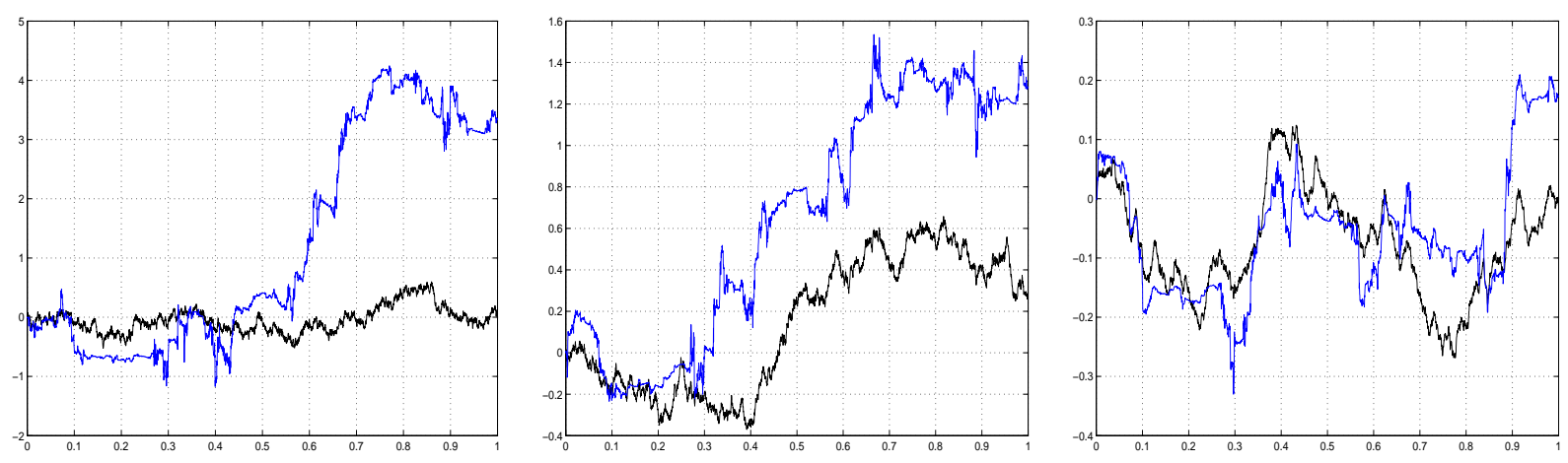

Figure 3: Sample paths of MRW. Sample path of $\widetilde{Z}^{\kappa}$ (blue-gray) together with that of $B^{\kappa}$ (black) from which it is obtained by combination with the sample path of $Q_{r}$ shown in Fig. 2. From left to right: $\kappa<0<\kappa_{c}, 0<\kappa<\kappa_{c}, 0<\kappa_{c}<\kappa$.
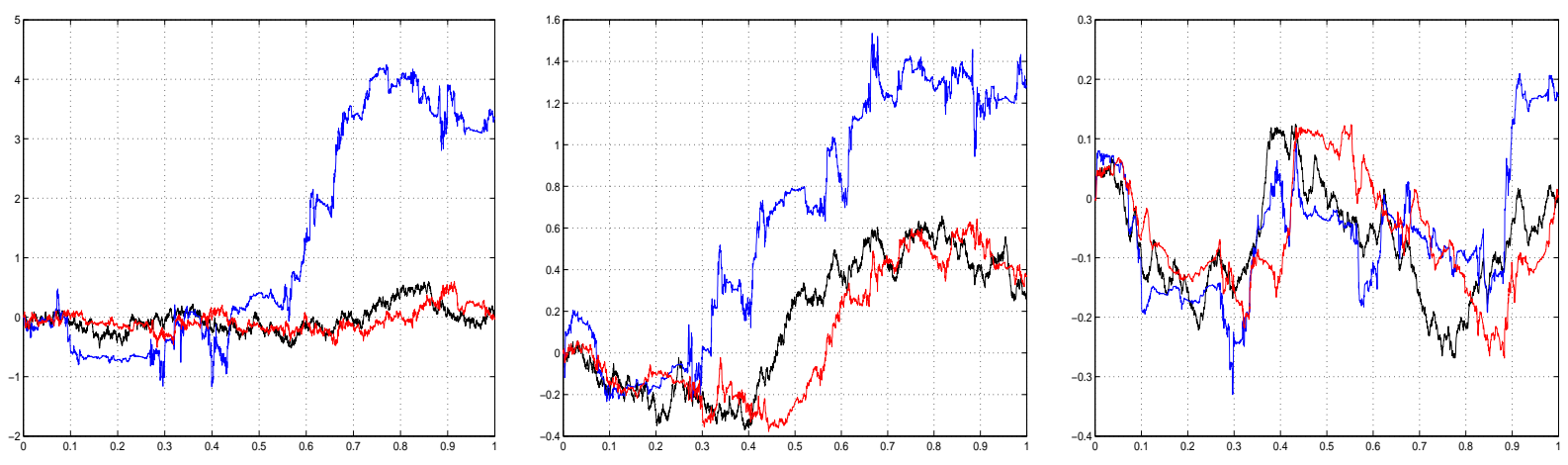

Figure 4: Superimposition of MRW and fBm in multifractal time. Using the sample paths of $Q_{r}$ shown in Fig. 2, superimpositions of the sample paths of $B^{\kappa}$ (black), $\widetilde{Z}^{\kappa}$ (blue-gray), $V^{\kappa}$ (red-light gray). From left to right: $\kappa<0<\kappa_{c}, 0<\kappa<\kappa_{c}, 0<\kappa_{c}<\kappa$. 


\subsection{Stationary increments and long range dependence}

In case $(A)$, MRW $\widetilde{Z}^{\kappa}$ has strictly stationary increments because $Q_{r}(u)$ is stationary and $B^{\kappa}(u)$ has stationary increments. The time series $X_{k}=\widetilde{Z}^{\kappa}((k+1) \tau)-\widetilde{Z}^{\kappa}(k \tau), k \in \mathbb{Z}$, for some $\tau>0$, of the increments of MRW $\widetilde{Z}^{\kappa}$ is therefore stationary. Similarly, in cases $(E)$ and $(B)$, MRW $\widetilde{Z}^{\kappa}$ has second order stationary increments and the series $X_{k}$ is second order stationary. The following result contains basic properties of $\widetilde{Z}^{\kappa}$ and $X_{k}$. See Section 5 for a proof.

Proposition 4.1 (i) In case $(A), \widetilde{Z}^{\kappa}$ has zero mean and the covariance structure

$$
E \widetilde{Z}^{\kappa}(s) \widetilde{Z}^{\kappa}(t)=C_{\kappa} \int_{0}^{s} \int_{0}^{t} E Q_{0}(u) Q_{0}(v)|u-v|^{2 \kappa-1} d u d v
$$

where $E Q_{0}(u) Q_{0}(v)$ is understood formally through (2.10), (5.1) and (5.2) by substituting $r=0$. In particular, for $k \geq 1 / \tau+1$,

$$
E X_{k} X_{0}=\frac{C_{\kappa} \tau^{2 \kappa+1}}{2 \kappa(2 \kappa+1)}\left(|k+1|^{2 \kappa+1}+|k-1|^{2 \kappa+1}-2|k|^{2 \kappa+1}\right) \sim C_{\kappa} \tau^{2 \kappa+1}|k|^{2 \kappa-1},
$$

as $k \rightarrow \infty$.

(ii) In cases $(E)$ and $(B), \widetilde{Z}^{\kappa}$ has the covariance

$$
E \widetilde{Z}^{\kappa}(s) \widetilde{Z}^{\kappa}(t)=C s, \quad \text { for } 0 \leq s \leq t .
$$

In particular, $E X_{k} X_{0}=0$, for $k \neq 0$. For example, in case (2.15), the constant in (4.3) is given by

$$
C=2 C_{\kappa}\left(-\frac{1}{c \varphi(2)+2 \kappa}+e^{-c \varphi(2)} \int_{0}^{1} d w w^{2 \kappa-1} e^{c \varphi(2) w}\right) .
$$

Time series $X_{k}$ with the autocovariance function satisfying (4.2) with $-1<2 \kappa-1<0$ as $k \rightarrow \infty$ is known as long range dependent. Thus, according to Proposition $4.1, \widetilde{Z}^{\kappa}$ is characterized only by two regimes at large scales: its increments are long range dependent for $\kappa_{c}<\kappa<1 / 2$ while it has uncorrelated increments for $0 \leq \kappa \leq \kappa_{c}$.

\subsection{Finiteness of moments}

The following result characterizes finiteness of even order moments of MRWs.

Proposition 4.2 Let $p \in \mathbb{N}$. In cases $(A),(E)$ and $(B)$, the $(2 p)$ th moment $E\left|\widetilde{Z}^{\kappa}(t)\right|^{2 p}$ is finite when

$$
\zeta(2 p)>1
$$

where $\zeta(q)$ is defined in (2.39).

The function $\zeta(q)$ plays the role of scaling exponents below. The condition (4.5) is therefore a natural and expected condition on finiteness of moments in the multifractal literature. In case $(A)$, the condition (4.5) was conjectured by Ludeña (2008).

\subsection{The special case of exact scale invariance}

The case of exact scale invariance (2.15) is special in the sense that the corresponding MRW has the following scaling property. 
Proposition 4.3 In the case of exact scale invariance, we have, for $a \in(0,1)$,

$$
\left\{\widetilde{Z}^{\kappa}(a t)\right\}_{t \in[0,1]} \stackrel{d}{=} a^{\kappa+\frac{1}{2}} e^{\Omega_{a}}\left\{\widetilde{Z}^{\kappa}(t)\right\}_{t \in[0,1]}
$$

in case $(A)$ with $\stackrel{d}{=}$ denoting the equality in the sense of finite dimensional distributions, and

$$
\left\{\widetilde{Z}^{\kappa}(a t)\right\}_{t \in[0,1]} \stackrel{m}{=} a^{\kappa_{c}+\frac{1}{2}} e^{\Omega_{a}}\left\{\widetilde{Z}^{\kappa}(t)\right\}_{t \in[0,1]}
$$

in cases $(E)$ and $(B)$, with $\stackrel{m}{=}$ denoting the equality of even order moments (from the set (3.6)) of linear combinations of finite dimensional distributions. In (4.6) and (4.7), $\Omega_{a}$ is a random variable independent of $\left\{\widetilde{Z}^{\kappa}(t)\right\}_{t \in[0,1]}$ and with the moment generating function (2.17).

Proof: In case $(A)$, observe from $(2.16)$ and $\left(\kappa+\frac{1}{2}\right)$-self-similarity of fBm that

$$
\widetilde{Z}_{a r}^{\kappa}(a t)=\int_{0}^{a t} Q_{a r}(u) d B^{\kappa}(u) \stackrel{d}{=} \int_{0}^{t} Q_{a r}(a x) d B^{\kappa}(a x) \stackrel{d}{=} a^{\kappa+\frac{1}{2}} e^{\Omega_{a}} \int_{0}^{t} Q_{r}(x) d B^{\kappa}(x) .
$$

The result (4.6) follows by letting $r \rightarrow 0$.

In case $(B)$, we have

$$
(a r)^{-(c \varphi(2)+2 \kappa) / 2} \int_{0}^{a t} Q_{a r}(u) d B^{\kappa}(u) \stackrel{m}{=} a^{-(c \varphi(2)+2 \kappa) / 2} a^{\kappa+\frac{1}{2}} e^{\Omega_{a}} r^{-(c \varphi(2)+2 \kappa) / 2} \int_{0}^{t} Q_{r}(x) d B^{\kappa}(x) .
$$

The result (4.7) follows from Proposition 3.1 by letting $r \rightarrow 0$. The same relation can also be deduced similarly in case $(E)$.

The relation (4.6) differs from the usual self-similarity in the presence of random variables $e^{\Omega_{a}}$. Proposition 4.3 above directly yields the scaling behavior of moments of MRWs (see Section 4.4.1).

\subsection{Scaling properties of moments of MRWs}

We study here the scaling properties of moments of MRW $\widetilde{Z}^{\kappa}$. These concern the asymptotic behavior of the moments $E\left|\widetilde{Z}^{\kappa}(t)\right|^{q}$ as $t \rightarrow 0$.

\subsubsection{The case of exact scale invariance}

A particularly easy case is that of exact scale invariance (2.15). Taking $a=t<1$ and $t=1$ in (4.6), yields $E\left|\widetilde{Z}^{\kappa}(t)\right|^{q}=t^{\left(\kappa+\frac{1}{2}\right) q} E e^{q \Omega_{t}} E|\widetilde{Z}(1)|^{q}=t^{(\kappa+1 / 2) q+c \varphi(q)} E|\widetilde{Z}(1)|^{q}$ in case $(A)$. In cases $(E)$ and (B), relation (4.7) yields $E\left|\widetilde{Z}^{\kappa}(t)\right|^{q}=t^{\left(\kappa_{c}+1 / 2\right) q+c \varphi(q)} E|\widetilde{Z}(1)|^{q}$ for even order moments $q$. Combining the two relations gives the following result.

Theorem 4.1 In the case of exact scale invariance (2.15) and $(A),(E)$ and $(B)$, we have, for $t \in(0,1)$,

$$
E\left|\widetilde{Z}^{\kappa}(t)\right|^{q}=C_{q} t^{\zeta(q)},
$$

where $\zeta(q)$ is defined in (2.39), and $q>0$ is such that $E\left|\widetilde{Z}^{\kappa}(1)\right|^{q}<\infty$.

The condition $E\left|\widetilde{Z}^{\kappa}(1)\right|^{q}<\infty$ holds for all even $q=2 p$ in $\mathcal{M}$ defined by (3.6). Note also that, in cases $(E)$ and $(B)$, scaling exponents $\zeta(q)$ depend only on $\kappa_{c}$, not $\kappa$.

Remark 4.1 In case $(A)$, note that (4.8) implies, in particular, $\left.E \mid \widetilde{Z}^{\kappa}(t)\right\rfloor^{2}=C_{2} t^{2 \kappa+1+c \varphi(2)}$, as $t \rightarrow 0$. On the other hand, as $t \rightarrow \infty$, it follows from (4.1) that $E\left|\widetilde{Z}^{\kappa}(t)\right|^{2} \sim \widetilde{C}_{2} t^{2 \kappa+1}$. The second moment thus scales differently at small and large scales. Note also that this is expected since small scales are influenced by $Q_{r}$ while larger scales are dominated by fBm. 


\subsubsection{The case of non-exact scale invariance}

In the case of non-exact scale invariance (2.14), the moments of MRW do not scale exactly. However, the scaling (4.8) is expected as $t \rightarrow 0$. We provide here such a result adapting the approach in Theorem 1 of Chainais et al. (2005a), p. 1069, which concerns the scaling properties of IDC motion. (Another possibility is to follow the approach used for Lemma 4 in Bacry and Muzy (2003).) Recall the notation $Q_{r}^{s}(u)$ defined in (2.7), and let $q>0$ be fixed. For $0<b<1, t \in(0,1]$ and $k \in \mathbb{N}$, let also

$$
\tau_{b, q}^{(k)}(t)=\left(E Q_{b^{k}}^{b^{k-1}}(0)^{q}\right)^{-1} E \sup _{0 \leq u, v \leq b^{k} t}\left|Q_{b^{k}}^{b^{k-1}}(u)^{q / 2} Q_{b^{k}}^{b^{k-1}}(v)^{q / 2}-Q_{b^{k}}^{b^{k-1}}(0)^{q}\right| .
$$

Theorem 4.2 Suppose $\kappa \in(0,1 / 2)$ and consider the case of scale invariance (2.14). Let $q>0$ be fixed. Suppose that, for $t \in(0,1]$,

$$
E\left|\widetilde{Z}_{r}^{\kappa}(t)\right|^{q} \rightarrow E\left|\widetilde{Z}^{\kappa}(t)\right|^{q}<\infty, \quad \text { as } r \rightarrow 0,
$$

and that, for $t \in(0,1]$ and $k \in \mathbb{N}$,

$$
\tau_{b, q}^{(k)}(t) \leq C_{b, q} t^{\nu}
$$

with $\nu>0$. Then, for $t \in(0,1]$ and some constants $\underline{C}_{q}, \bar{C}_{q}$,

$$
\underline{C}_{q} t^{\zeta(q)} \leq E\left|\widetilde{Z}^{\kappa}(t)\right|^{q} \leq \bar{C}_{q} t^{\zeta(q)}
$$

where $\zeta(q)$ is defined in (2.39).

See Section 5 for a proof of the theorem. In regard to the assumption (4.11), observe that, by the Hölder's inequality and the inequality $|a-b|^{2} \leq\left|a^{2}-b^{2}\right|, a, b>0$,

$$
\begin{gathered}
E \sup _{0 \leq u, v \leq b^{k} t}\left|Q_{b^{k}}^{b^{k-1}}(u)^{q / 2} Q_{b^{k}}^{b^{k-1}}(v)^{q / 2}-Q_{b^{k}}^{b^{k-1}}(0)^{q}\right| \\
\leq E \sup _{0 \leq u \leq b^{k} t} Q_{b^{k}}^{b^{k-1}}(u)^{q / 2} \sup _{0 \leq v \leq b^{k} t}\left|Q_{b^{k}}^{b^{k-1}}(v)^{q / 2}-Q_{b^{k}}^{b^{k-1}}(0)^{q / 2}\right| \\
+E Q_{b^{k}}^{b^{k-1}}(0)^{q / 2} \sup _{0 \leq u \leq b^{k} t}\left|Q_{b^{k}}^{b^{k-1}}(u)^{q / 2}-Q_{b^{k}}^{b^{k-1}}(0)^{q / 2}\right| \\
\leq\left\{\left(E \sup _{0 \leq u \leq b^{k} t} Q_{b^{k}}^{b^{k-1}}(u)^{q}\right)^{1 / 2}+\left(E Q_{b^{k}}^{b^{k-1}}(0)^{q}\right)^{1 / 2}\right\}\left\{E \sup _{0 \leq u \leq b^{k} t}\left|Q_{b^{k}}^{b^{k-1}}(u)^{q}-Q_{b^{k}}^{b^{k-1}}(0)^{q}\right|\right\}^{1 / 2} .
\end{gathered}
$$

Hence,

$$
\begin{aligned}
\tau_{b, q}^{(k)}(t) & \leq\left\{\left(\frac{E \sup _{0 \leq u \leq b^{k} t} Q_{b^{k}}^{b^{k-1}}(u)^{q}}{E Q_{b^{k}}^{b^{k-1}}(0)^{q}}\right)^{1 / 2}+1\right\}\left\{\frac{E \sup _{0 \leq u \leq b^{k} t}\left|Q_{b^{k}}^{b^{k-1}}(u)^{q}-Q_{b^{k}}^{b^{k-1}}(0)^{q}\right|}{E Q_{b^{k}}^{b^{k-1}}(0)^{q}}\right\}^{1 / 2} \\
& =:\left\{\left(\frac{E \sup _{0 \leq u \leq b^{k} t} Q_{b^{k}}^{b^{k-1}}(u)^{q}}{E Q_{b^{k}}^{b^{k-1}}(0)^{q}}\right)^{1 / 2}+1\right\} \widetilde{\tau}_{b, q}^{(k)}(t) \leq\left(\left(\widetilde{\tau}_{b, q}^{(k)}(t)^{2}+1\right)^{1 / 2}+1\right) \widetilde{\tau}_{b, q}^{(k)}(t) .
\end{aligned}
$$

The functions $\widetilde{\tau}_{b, q}^{(k)}(t)$ are used in Chainais et al. (2005a) in the assumption similar to the assumption (4.11). Sufficient conditions for these functions to be bounded by the function $C_{b, q} t^{\nu}, \nu>0$, are provided (see Corollary 2 in Chainais et al. (2005a)). 
Remark 4.2 When $0 \leq \kappa \leq \kappa_{c}$ (cases $(E)$ and $(B)$ ), the scaling behavior of MRWs is the same according to Theorems 4.1 and 4.2. In particular, from a practical perspective, this suggests that the MRW with $\kappa=0<\kappa_{c}$ could be used to capture the corresponding scaling behavior. The case $\kappa=0$ is obviously simpler than that of $\kappa \neq 0$.

Remark 4.3 As in Chainais et al. (2005a), Theorem 4.2 can be reformulated for control measures $m$ that are more general than in (2.14). In this case, the term $t^{\zeta(q)}$ in (4.12) gets replaced by $t^{\left(\kappa^{*}+\frac{1}{2}\right) q} e^{-\varphi(q) m\left(C_{t}(0)\right)}$, where $\kappa^{*}$ is defined in $(2.40)$.

\subsection{Numerical illustrations}

We now intend to illustrate practically the scaling behaviors reported in (4.8) and (4.12). These power law behaviors of $\widetilde{Z}^{\kappa}$ translate into those of its stationary increments as

$$
E\left|\widetilde{Z}^{\kappa}\left(t+a \tau_{0}\right)-\widetilde{Z}^{\kappa}(t)\right|^{q}=C_{q}^{\prime}|a|^{\zeta(q)},
$$

for $q>0$, such that $E\left|\widetilde{Z}^{\kappa}(1)\right|^{q}<\infty$, where $\tau_{0}$ is an arbitrary constant, and $a$ is an analysis scale such that $a \tau_{0} \leq 1$. The scaling exponents $\zeta(q)$ are given in (2.39). To reproduce such behaviors from a single realization of $\widetilde{Z}^{\kappa}(t)$, one usually replaces the ensemble averages with time averages, called structure functions,

$$
S(a, q)=\frac{1}{n_{a}} \sum_{k=0}^{n_{a}}\left|\widetilde{Z}^{\kappa}\left(k a \tau_{0}+a \tau_{0}\right)-\widetilde{Z}^{\kappa}\left(k a \tau_{0}\right)\right|^{q}
$$

where $n_{a}$ is the number of such increments available at scale $a$. The estimation $\hat{\zeta}(q)$ of the scaling exponents $\zeta(q)$ is obtained by performing a weighted linear regression in a $\log _{2} 2^{j}$ against $\log _{2} S\left(2^{j}, q\right)$ diagram. This procedure is fully defined in, for example, Lashermes et al. (2004).

However, it is of importance to mention that, as now commonly agreed, the time averages $S(a, q)$ reproduce the power law behaviors $S(a, q) \sim C^{\prime \prime}(q) a^{\zeta(q)}$, implied by (4.13) above, only over a finite range of $q \in\left[0, q_{*}^{+}\right]$, where $q_{*}^{+}$is defined as the value of $q>1$ such that $1+q \zeta^{\prime}(q)-\zeta(q)=0$. This is detailed in Lashermes et al. (2004).

Fig. 5 shows the mean values of the estimates $\hat{\zeta}(q)$ for $q \in\left[0, q_{*}^{+}\right]$obtained from averages over 500 realizations of sample paths of $\widetilde{Z}^{\kappa}$ (with $N=2^{15}$ and $R=2^{4}$ ). As earlier, we take $\kappa_{c}=0.12$. Results illustrated in Fig. 5 clearly indicate that the estimated scaling exponents $\hat{\zeta}(q)$ are in fairly satisfactory agreement with the predicted values $\zeta(q)$ when $0<\kappa_{c}<\kappa$ (case $\left.(A)\right)$ and $0<\kappa<\kappa_{c}$ (case $(B)$ ). They also show that this agreement is less satisfactory when $\kappa<0<\kappa_{c}$ (case $(N)$ ). However, the agreement tends to improve when the oversampling rate $R$ is increased, at the price, though, of a significant increase in the computational cost and computer memory issues. This clearly indicates that the use of Riemann sums to approximate the continuous sum defining $Z^{\kappa}$ is less efficient when $\kappa<0$ (compared to when $\kappa>0$ ).

The numerical analysis described above can be straightforwardly extended to structure functions $S^{L}\left(2^{j}, q\right)$ obtained by replacing increments at scale $a=2^{j}$ by wavelet leaders. Wavelet leaders are defined as local suprema of the discrete wavelet transform coefficients of $\widetilde{Z}^{\kappa}$ (see Jaffard et al. (2004, 2006) for definitions, implementations and performance). Such structure functions can be computed both for positive and negative values of $q \in\left[q_{*}^{-}, q_{*}^{+}\right]$, where $q_{*}^{-}$and $q_{*}^{+}$are the negative and positive zeros of $1+q \zeta^{\prime}(q)-\zeta(q)=0$. For multifractal processes, the structure functions $S^{L}\left(2^{j}, q\right)$ exhibit power law behaviors in the limit of fine scales $S^{L}\left(2^{j}, q\right) \sim c_{q} 2^{j \zeta^{L}(q)}$ and a Legendre transform of $\zeta^{L}(q)$ provides a tight upper bound of the multifractal spectrum $D(h)$ of the analyzed process: $D(h) \leq \min _{q \neq 0}\left(1+q h-\zeta^{L}(q)\right)$ (cf. Jaffard (2004)). The $\zeta^{L}(q)$ are estimated via a weighted linear fit as above.

Results (cf. Fig. 6) obtained from 500 realizations of $\widetilde{Z}^{\kappa}$ (with $N=2^{15}$ and $R=2^{4}$ ) show a very satisfactory agreements between the estimated scaling exponents $\hat{\zeta}^{L}(q)$ and the theoretical exponents 

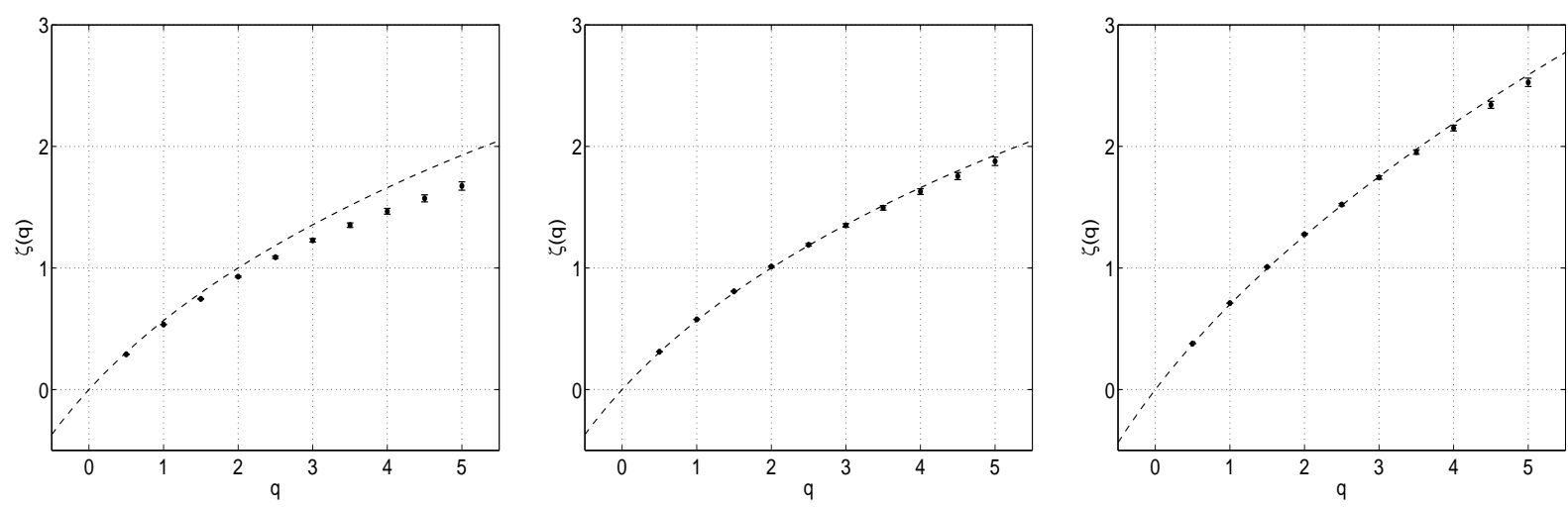

Figure 5: Scaling exponents from increments. For $\kappa=-0.1<0,0<\kappa=0.02<\kappa_{c}$ and $0<$ $\kappa_{c}<\kappa=0.2$ (from left to right), dashed lines: theoretical scaling exponents; solid points: scaling exponents estimated using increment based structure functions; for $q \in\left[0, q_{*}^{+}\right]$. The agreement is very satisfactory when $\kappa>0$. The case $\kappa<0$ is more difficult, requiring the use of higher oversampling rate $R$, and hence higher practical difficulties (computational cost and memory issues).
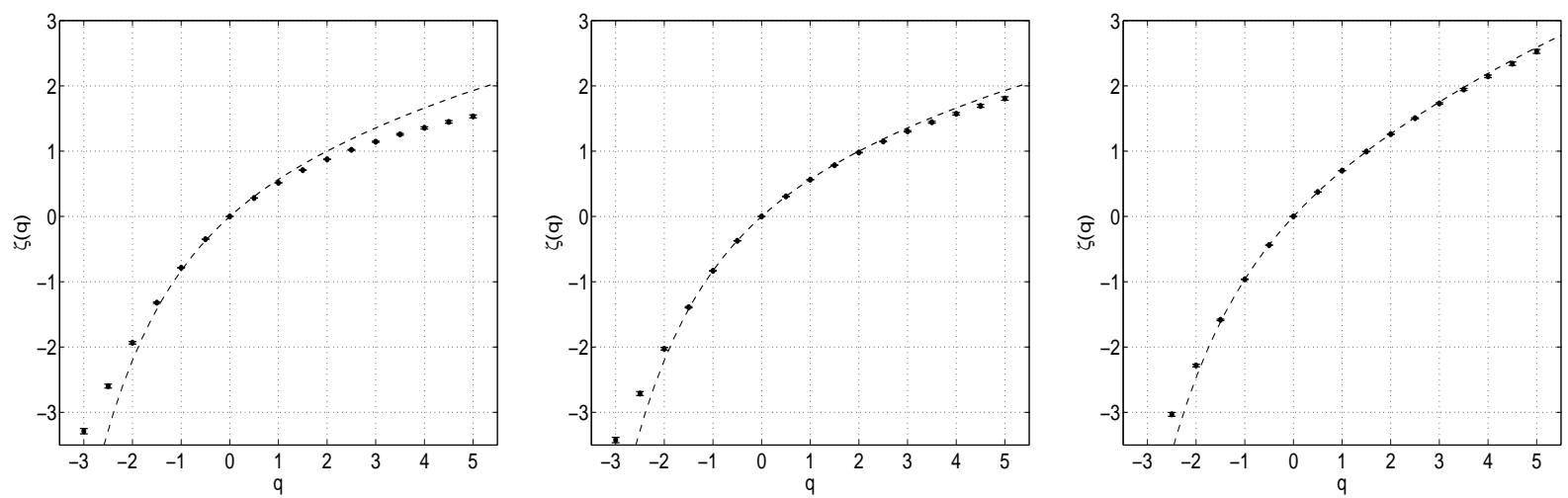

Figure 6: Scaling exponents from wavelet leaders. For $\kappa=-0.1<0,0<\kappa=0.02<\kappa_{c}$ and $0<\kappa_{c}<\kappa=0.2$ (from left to right), dashed lines: theoretical scaling exponents; solid points: scaling exponents estimated using wavelet leader based structure functions; for $q \in\left[q_{*}^{-}, q_{*}^{+}\right]$. The agreement is very satisfactory when $\kappa>0$ for both positive and negative $q$ while slightly less when $\kappa<0$. 
$\zeta(q)$ given in (2.39) over the full range $q \in\left[q_{*}^{-}, q_{*}^{+}\right]$, i.e., including positive and negative values of $q$, when $0<\kappa_{c}<\kappa($ case $(A))$ and $0<\kappa<\kappa_{c}$ (case $(B)$ ). For the case $\kappa<0$ (case $(N)$ ), a discrepancy is visible, indicating the requirement to use higher oversampling factors.

\subsection{MRW versus subordinated FBM}

In this closing subsection, we compare MRW $Z^{\kappa}$ and $\mathrm{fBm}$ in multifractal time $V^{\kappa}$ as defined in (3.18). We are interested in whether these processes can have the same scaling exponents and, more generally, the same finite dimensional distributions. The answer turns out to depend on whether the processes $Z^{\kappa}$ and $V^{\kappa}$ are defined using:

Case 1: same parameter $\kappa$ and function $\varphi$;

Case 2: same function $\varphi$ but different $\kappa$;

Case 3: same $\kappa$ but different functions $\varphi$;

Case 4: different parameters $\kappa$ and functions $\varphi$.

In Cases 1, 2 and $3, Z^{\kappa}$ and $V^{\kappa}$ essentially have different scaling exponents, and hence different finite dimensional distributions. In Case 4, these processes can have the same scaling exponents. Whether their finite dimensional distributions are the same is a more challenging issue and will be explored elsewhere. Below is a more detailed treatment of these cases.

Case 1: In this case, the scaling exponents of MRW $Z^{\kappa}$ and fBm in multifractal time $V^{\kappa}$ are

$$
\zeta_{V}(q)=(\kappa+1 / 2) q+c \varphi(q(\kappa+1 / 2)), \quad \zeta_{Z}(q)=\left(\kappa^{*}+1 / 2\right) q+c \varphi(q)
$$

where $\kappa^{*}$ is defined in (2.40). When $\kappa^{*}=\kappa$, the scaling exponents are clearly different. When $\kappa^{*}=-\varphi(2) / 2$, the same conclusion can be drawn under a mild assumption of twice differentiability of $\varphi(q)$ at $q=0$. Indeed, if $\zeta_{Z}(q)=\zeta_{V}(q)$, then $\zeta_{Z}^{\prime \prime}(0)=c(\kappa+1 / 2)^{2} \varphi^{\prime \prime}(0)$ and $\zeta_{Y}^{\prime \prime}(0)=c \varphi^{\prime \prime}(0)$ have to be equal, which leads to contradiction. If the scaling exponents are different, the corresponding finite dimensional distributions are different as well.

Case 2: This case can be dealt with in the same way as Case 1 assuming twice differentiability of $\varphi(q)$ at $q=0$.

Case 3: In this case, the corresponding scaling exponents are

$$
\zeta_{V}(q)=(\kappa+1 / 2) q+c \varphi_{1}(q(\kappa+1 / 2)), \quad \zeta_{Z}(q)=\left(\kappa^{*}+1 / 2\right) q+c \varphi_{2}(q),
$$

where $\kappa^{*}=\max \left\{\kappa, \kappa_{2, c}\right\}, \kappa_{2, c}=-\varphi_{2}(2) / 2$. A particular class of functions $\varphi_{2}$ are

$$
\varphi_{2}(q)=\varphi_{1}(\beta q)-q \varphi_{1}(\beta),
$$

for some $\beta>0$, which are transformations arising in (3.16). To understand whether the equality of $\zeta_{Z}$ and $\zeta_{V}$ is possible, several subcases need to be considered.

- If $\kappa \geq \kappa_{2, c}$, then $\kappa^{*}=\kappa$ and to have $\zeta_{V}(q)=\zeta_{Z}(q)$, we need $\varphi_{1}(q(\kappa+1 / 2))=\varphi_{2}(q)$. This is not possible given basic properties of $\varphi_{i}, i=1,2\left(\varphi_{i}(0)=\varphi_{i}(1)=0\right.$, concavity).

- If $\kappa<\kappa_{2, c}$, on the other hand, then $\kappa^{*}=-\varphi_{2}(2) / 2$ and $\zeta_{V}(q)=\zeta_{Z}(q)$ becomes

$$
(\kappa+1 / 2) q+c \varphi_{1}(q(\kappa+1 / 2))=\left(-\varphi_{2}(2) / 2+1 / 2\right) q+c \varphi_{2}(q) .
$$


This yields, in particular,

$$
\zeta_{V}(2)=(\kappa+1 / 2) 2+c \varphi_{1}(2(\kappa+1 / 2))=\left(-\varphi_{2}(2) / 2+1 / 2\right) 2+c \varphi_{2}(2)=1 .
$$

If $\zeta_{\mathrm{IDC}}(q)=q+c \varphi_{1}(q)$ are the scaling exponents (2.21) of the IDC motion, the equality (4.18) implies that

$$
\zeta_{\mathrm{IDC}}(2(\kappa+1 / 2))=1 .
$$

If $\kappa \in(-1 / 2,0)$, the relation (4.19) shows that $\zeta_{\text {IDC }}\left(q_{0}\right)=1$ for some $q_{0} \in(0,1)$. By concavity, one expects that $\zeta_{\text {IDC }}^{\prime}(1)<0$ and this situation corresponds to the case when the IDC motion and hence fBm in multifractal time are degenerate (Bacry and Muzy (2003)).

If $\kappa \in\left(0, \kappa_{2, c}\right)$, (4.19) means that $\zeta_{\mathrm{IDC}}\left(q_{0}\right)=1$ for some $q_{0} \in(1,2)$. This corresponds to another unusual situation when the IDC motion is expected to have infinite variance.

When $\kappa=0$, no such conclusion can be drawn. In fact, when $\kappa=0,(4.17)$ is

$$
q / 2+c \varphi_{1}(q / 2)=\left(-\varphi_{2}(2) / 2+1 / 2\right) q+c \varphi_{2}(q)
$$

or, after noticing that $\varphi_{1}(1 / 2)=-\varphi_{2}(2) / 2$,

$$
\varphi_{2}(q)=\varphi_{1}(q / 2)-q \varphi_{1}(1 / 2) / 2,
$$

which is the transformation (4.16) with $\beta=1 / 2$. This choice corresponds to using $\varphi_{1}$ and $\beta=1 / 2$ as a power in the definition (3.14) for MRW. As discussed in Section 3.2.3, the resulting MRW and $\mathrm{fBm}$ in multifractal time have the same finite dimensional distributions.

Case 4: In this case, the corresponding scaling exponents are

$$
\zeta_{V}(q)=\left(\kappa_{1}+1 / 2\right) q+c \varphi_{1}\left(q\left(\kappa_{1}+1 / 2\right)\right), \quad \zeta_{Z}(q)=\left(\kappa_{2}^{*}+1 / 2\right) q+c \varphi_{2}(q)
$$

where $\kappa_{2}^{*}=\max \left\{\kappa_{2}, \kappa_{2, c}\right\}, \kappa_{2, c}=-\varphi_{2}(2) / 2$. The equality of scaling exponents, in fact, is possible here even in the case when $\kappa_{2}^{*}=\kappa_{2}$, that is, $\kappa_{2} \geq-\varphi_{2}(2) / 2$. Indeed, from a theoretical standpoint, it is just enough to take

$$
\begin{aligned}
\kappa_{2} & =\kappa_{1}+\varphi_{1}\left(\kappa_{1}+1 / 2\right) \\
\varphi_{2}(q) & =\varphi_{1}\left(q\left(\kappa_{1}+1 / 2\right)\right)-q \varphi_{1}\left(\kappa_{1}+1 / 2\right) .
\end{aligned}
$$

The following example illustrates that the choice (4.23)-(4.24) is legitimate even in the class of log normal cascades.

Example 4.1 Consider log normal IDCs with

$$
\varphi_{i}(q)=\frac{\sigma_{i}^{2}}{2}\left(q-q^{2}\right), \quad i=1,2
$$

After elementary calculations, the choice (4.23)-(4.24) translates into

$$
\sigma_{2}=H_{1} \sigma_{1}, \quad H_{2}=H_{1}+\frac{\sigma_{1}^{2}}{2}\left(H_{1}-H_{1}^{2}\right),
$$

where we set $H_{i}=\kappa_{i}+1 / 2$ as usual. Note that using (4.26), the condition $\kappa_{2}>-\varphi_{2}(2) / 2$ is equivalent to

$$
\frac{1}{\sigma_{1}^{2}}>H_{1}
$$


given that $H_{1}>1 / 2$. On the other hand, if at least two moments are expected finite for the corresponding IDC motion, it is required (Bacry and Muzy (2003)) that

$$
\frac{1}{\sigma_{1}^{2}}>1
$$

The latter condition implies (4.27). Finally, note similarly that $H_{2}<1$ is equivalent to

$$
\frac{2}{\sigma_{1}^{2}}>1
$$

which is even weaker than (4.27).

Though the choice (4.23)-(4.24) yields the same scaling exponents, we expect that the corresponding finite dimensional distributions of the processes are different. Showing this here, however, would take us beyond the scope of the paper. Just to indicate some of the difficulties, note that the equality of scaling exponents for $q=2$ and stationarity of the increments implies that the two processes have the same second order properties. In order to show that their finite dimensional distributions are different, higher order properties at several time points (for example, $E X(s)^{2} X(t)^{2}$ where $X$ is either MRW or fBm in multifractal time) need to be considered. The latter properties go beyond typical multifractal analysis, and will be studied elsewhere.

\section{Proofs of auxiliary results}

Throughout this section, we shall use the following expressions for the function $m_{r}(u)$ defined in (2.11). In the cases (2.14) and (2.15), one has

$$
\begin{gathered}
m_{r}(u)=\left\{\begin{array}{cl}
0, & \text { if } u>1, \\
-c \ln u+c(u-1), & \text { if } r<u \leq 1, \\
-c \ln r+c u\left(1-\frac{1}{r}\right) & \text { if } 0 \leq u \leq r,
\end{array}\right. \\
m_{r}(u)=\left\{\begin{array}{cl}
0, & \text { if } u>1, \\
-c \ln u, & \text { if } r<u \leq 1, \\
-c \ln r+c\left(1-\frac{u}{r}\right), & \text { if } 0 \leq u \leq r,
\end{array}\right.
\end{gathered}
$$

respectively.

Proof of Proposition 2.1: Suppose for simplicity that $t=1$. For $\kappa \in(0,1 / 2)$, since $Q_{r}(u)$ is nonnegative, it is enough to show that $E \int_{0}^{1} \int_{0}^{1} Q_{r}(u) Q_{r}(v)|u-v|^{2 \kappa-1} d u d v<\infty$. This follows from $E Q_{r}(u) Q_{r}(v) \leq C_{r}<\infty$ for all $u, v \in[0,1]$, using (2.10) and (5.1), (5.2). For $\kappa \in(-1 / 2,0)$, using Corollary 2.1, it is enough to show that $I_{1}<\infty$ and $I_{2}<\infty$, where

$$
\begin{gathered}
I_{1}=E \int_{0}^{1}\left(Q_{r}(u)(t-u)^{\kappa}\right)^{2} d u \\
I_{2}=E \int_{0}^{1} u^{-2 \kappa}\left(\int_{0}^{1}\left|s^{\kappa} Q_{r}(s)-u^{\kappa} Q_{r}(u)\right|(u-s)_{+}^{\kappa-1} d s\right)^{2} d u .
\end{gathered}
$$

The case of $I_{1}$ is immediate since $E Q_{r}(u)^{2}=C_{r}<\infty$ and $2 \kappa+1>0$. For $I_{2}$, by the generalized Minkowski's inequality, it is enough to show that $J_{1}<\infty$ and $J_{2}<\infty$, where

$$
J_{1}=\int_{0}^{r} u^{-2 \kappa}\left(\int_{0}^{u}\left(E\left(s^{\kappa} Q_{r}(s)-u^{\kappa} Q_{r}(u)\right)^{2}\right)^{1 / 2}(u-s)^{\kappa-1} d s\right)^{2} d u
$$




$$
J_{2}=\int_{r}^{1} u^{-2 \kappa}\left(\int_{u-r}^{u}\left(E\left(s^{\kappa} Q_{r}(s)-u^{\kappa} Q_{r}(u)\right)^{2}\right)^{1 / 2}(u-s)^{\kappa-1} d s\right)^{2} d u
$$

Observe that

$$
E\left(s^{\kappa} Q_{r}(s)-u^{\kappa} Q_{r}(u)\right)^{2}=\left(s^{2 \kappa}+u^{2 \kappa}\right) e^{-\varphi(2) m_{r}(0)}-2 s^{\kappa} u^{\kappa} e^{-\varphi(2) m_{r}(u-s)},
$$

where $m_{r}(u)$ is defined in (2.11). Then, after a change of variables $s=u v$, write $J_{1}$ as

$$
J_{1}=C_{r} \int_{0}^{r} u^{-2 \kappa}\left(\int_{0}^{1}\left(\left(1+v^{2 \kappa}\right)-2 v^{2 \kappa} e^{-\varphi(2)\left(m_{r}(u-u v)-m_{r}(0)\right)}\right)^{1 / 2}(1-v)^{\kappa-1} d v\right)^{2} d u .
$$

We have $J_{1}<\infty$ if $J_{1,1}<\infty$ and $J_{1,2}<\infty$, where

$$
\begin{gathered}
J_{1,1}=\int_{0}^{r} u^{2 \kappa} d u\left(\int_{0}^{1}\left(\left(1+v^{2 \kappa}\right)-2 v^{2 \kappa}\right)^{1 / 2}(1-v)^{\kappa-1} d v\right)^{2} \\
J_{1,2}=\int_{0}^{r} u^{2 \kappa}\left(\int_{0}^{1}\left(m_{r}(u(1-v))-m_{r}(0)\right)^{1 / 2}(1-v)^{\kappa-1} d v\right)^{2} d u .
\end{gathered}
$$

Finiteness of $J_{1,1}$ follows from $\kappa \in(-1 / 2,1 / 2)$ and $\left(1+v^{2 \kappa}\right)-2 v^{2 \kappa} \sim C(1-v)$, as $v \uparrow 1$. Finiteness of $J_{1,2}$ follows from (5.1), (5.2) by observing that $m_{r}(0)-m_{r}(u(1-v)) \leq C_{r} u(1-v)$. This shows that $J_{1}<\infty$. The finiteness of $J_{2}$ can be proved in a similar way.

In the proof of Proposition 3.1 below, we shall use the following notation and facts. Let $\mathcal{P}_{2 p}$ consists of collections of pairs $\{(i, j)\}$ such that $i<j$, and

$$
(i, j) \cap\left(i^{\prime}, j^{\prime}\right)=\emptyset
$$

for two pairs $(i, j)$ and $\left(i^{\prime}, j^{\prime}\right)$ from the same collection, and

$$
\bigcup_{\{(i, j)\}}\{i, j\}=\{1,2, \ldots, 2 p\} .
$$

For example, $\mathcal{P}_{2}$ consists of $\{(1,2)\}$, and $\mathcal{P}_{4}$ consists of $\{(1,2),(3,4)\},\{(1,3),(2,4)\},\{(1,4),(2,3)\}$. Let also $\mathcal{P}_{2 p-1}$ consists of all pairs $\{(i, j)\}=\left\{\left(i^{\prime}, j^{\prime}\right)\right\} \backslash\left\{\left(i_{2 p}^{\prime}, 2 p\right)\right\}$ with $\left\{\left(i^{\prime}, j^{\prime}\right)\right\} \in \mathcal{P}_{2 p}$ and $\left(i_{2 p}^{\prime}, 2 p\right)$ indicating a pair containing $2 p$. For example, $\mathcal{P}_{3}$ consists of $\{(1,2)\},\{(1,3)\},\{(2,3)\}$. We shall also regularly use the fact that

$$
\sum_{2 \leq k \leq l \leq q} \alpha_{l-k+2}=\varphi(q)
$$

where $\alpha_{k}$ is defined in (2.13) (see also Eq. (21) in Bacry and Muzy (2003)).

Proof of Proposition 3.1: We suppose for simplicity that $t=1$ and consider only the case of exact scale invariance (2.15). (It is clear from the proof below that the arguments can also be adapted to the case (2.14).) Observe that, by conditioning on $Q_{r}$ and using (2.31) and (2.24),

$$
\begin{gathered}
E\left|Z_{r}^{\kappa}(1)\right|^{2 p}=C E\left(\int_{0}^{1} \int_{0}^{1} Q_{r}(u) Q_{r}(v)|u-v|^{2 \kappa-1} d u d v\right)^{p} \\
=C \int_{0}^{1} \int_{0}^{1} \ldots \int_{0}^{1} \int_{0}^{1} E Q_{r}\left(u_{1}\right) Q_{r}\left(v_{1}\right) \ldots Q_{r}\left(u_{p}\right) Q_{r}\left(v_{p}\right)\left|u_{1}-v_{1}\right|^{2 \kappa-1} \ldots\left|u_{p}-v_{p}\right|^{2 \kappa-1} d u_{1} d v_{1} \ldots d u_{p} d v_{p} .
\end{gathered}
$$

(Here and below, $C$ will denote a generic constant that may change from line to line.) By using (2.12), we further obtain (with the notation $\mathcal{P}_{2 p}$ above) that

$$
E\left|Z_{r}^{\kappa}(1)\right|^{2 p}=C \sum_{\{(i, j)\} \in \mathcal{P}_{2 p}} \int_{0<z_{1}<\ldots<z_{2 p}<1} d z_{1} \ldots d z_{2 p}
$$




$$
\exp \left\{-\sum_{1 \leq k<l \leq 2 p} \alpha_{l-k+1} m_{r}\left(z_{l}-z_{k}\right)\right\} \prod_{\{(i, j)\}}\left(z_{j}-z_{i}\right)^{2 \kappa-1} .
$$

Note that, after a change of variables $z_{1}=w_{1}, z_{k}-z_{k-1}=w_{k}, k=2, \ldots, 2 p$, we also have

$$
\begin{gathered}
E\left|Z_{r}^{\kappa}(1)\right|^{2 p} \leq C \sum_{\{(i, j)\} \in \mathcal{P}_{2 p}} \int_{0}^{1} \ldots \int_{0}^{1} d w_{2} \ldots d w_{2 p} \\
\exp \left\{-\sum_{2 \leq k \leq l \leq 2 p} \alpha_{l-k+2} m_{r}\left(w_{k}+\ldots+w_{l}\right)\right\} \prod_{\{(i, j)\}}\left(w_{i+1}+\ldots+w_{j}\right)^{2 \kappa-1}=: C I_{r}(2 p),
\end{gathered}
$$

where the inequality above is due to dropping the restrictions $w_{1}+\ldots+w_{k}<1, k=2, \ldots 2 p$. We will show that

$$
I_{r}(2 p) \sim C n(r)^{2 p}
$$

where $n(r)$ is given by (3.3). Since $E\left|Z_{r}^{\kappa}(1)\right|^{2 p}$ increases as $r \rightarrow 0$ in view of (5.7), this immediately yields (3.2) in case $(A)$. In cases $(E)$ and $(B)$, similar arguments to those below would show that the rate of increase of the difference between $I_{r}(2 p)$ and $E\left|Z_{r}^{\kappa}(1)\right|^{2 p}$ is slower than $n(r)^{2 p}$. Moreover, only the case $(B)$ will be considered. We prove $(5.8)$ for cases $(A)$ and $(B)$ separately.

Case $(A)$ : Since $m_{r}(u) \leq-c \ln u$ for all $u, r>0$, it is enough to show that $I(2 p)<\infty$, where

$$
I(q)=\int_{0}^{1} \ldots \int_{0}^{1} d w_{2} \ldots d w_{q} \prod_{2 \leq k \leq l \leq q}\left(w_{k}+\ldots+w_{l}\right)^{c \alpha_{l-k+2}} \prod_{\{(i, j)\}}\left(w_{i+1}+\ldots+w_{j}\right)^{2 \kappa-1}
$$

and $\{(i, j)\}$ is any fixed collection of pairs from $\mathcal{P}_{2 p}$. This will be achieved through induction in $p$ and we shall also use $I(q)$ for odd powers $q$ defined by (5.9) but where $\{(i, j)\} \in \mathcal{P}_{q}$.

When $p=1, I(2)=\int_{0}^{1} w_{2}^{c \alpha_{2}+2 \kappa-1} d w_{2}$ which is finite since $c \alpha_{2}+2 \kappa=c \varphi(2)+2 \kappa>0$ in case $(A)$. Suppose $I(k)<\infty, k=1, \ldots, q-1$, and consider the case of $I(q)$. (Note that, since $\varphi$ is concave, $(\kappa+1 / 2) 2+c \varphi(2)>1$ and $(\kappa+1 / 2) q+c \varphi(q)>1$ imply $(\kappa+1 / 2) k+c \varphi(k)>1$ for $k=2, \ldots, q$. Denote by $I_{n}(q), n=2, \ldots, q$, the integral $I(q)$ restricted to the set $w_{m}<w_{n}$, all $m \neq n$. It is then enough to show that

$$
I_{n}(q)<\infty, \quad n=2, \ldots, q .
$$

We need to consider the cases $q=2 p$ and $q=2 p-1$ separately.

Consider first the case of even $q=2 p$. Then, making a change of variables $w_{n}=s_{n}, w_{m}=s_{n} t_{m}$, $m \neq n$, and using the fact (5.5), we obtain that

$$
\begin{gathered}
I_{n}(2 p)=\int_{0}^{1} d s_{n} s_{n}^{(2 \kappa-1) p+2 p-2+c \varphi(2 p)} \\
\int_{0}^{1} \ldots \int_{0}^{1} d t_{2} \ldots d t_{n-1} d t_{n+1} \ldots d t_{2 p} \prod_{2 \leq k \leq l \leq 2 p}\left(t_{k}+\ldots+t_{l}\right)^{c \alpha_{l-k+2}} \prod_{\{(i, j)\}}\left(t_{i+1}+\ldots+t_{j}\right)^{2 \kappa-1},
\end{gathered}
$$

where $t_{n}=1$ by convention in the last multiple integral. Since $t_{n}=1$, we further have

$$
\begin{gathered}
I_{n}(2 p) \leq C \int_{0}^{1} d s_{n} s_{n}^{\zeta(2 p)-2} \\
\int_{0}^{1} \ldots \int_{0}^{1} d t_{2} \ldots d t_{n-1} \prod_{2 \leq k \leq l \leq n-1}\left(t_{k}+\ldots+t_{l}\right)^{c \alpha_{l-k+2}} \prod_{\left\{\left(i^{\prime}, j^{\prime}\right)\right\}}\left(t_{i^{\prime}+1}+\ldots+t_{j^{\prime}}\right)^{2 \kappa-1} \\
\int_{0}^{1} \ldots \int_{0}^{1} d t_{n+1} \ldots d t_{2 p} \prod_{2 \leq k \leq l \leq 2 p-(n-1)}\left(t_{n-1+k}+\ldots+t_{n-1+l}\right)^{c \alpha_{l-k+2}} \prod_{\left\{\left(i^{*}, j^{*}\right)\right\}}\left(t_{n+i^{*}}+\ldots+t_{n+j^{*}}\right)^{2 \kappa-1},
\end{gathered}
$$


where $\left\{\left(i^{\prime}, j^{\prime}\right)\right\}$ is a collection of pairs from $\mathcal{P}_{n-1}$ and $\left\{\left(i^{*}, j^{*}\right)\right\}$ is a collection of pairs from $\mathcal{P}_{2 p-(n-1)}$. These collections may not be defined uniquely and can be chosen only to make a convenient bound. For example, in the case $p=2,\{(i, j)\}=\{(2,3),(1,4)\}$ and $n=3, I_{3}(4)$ in $(5.11)$ is

$$
I_{3}(4)=\int_{0}^{1} d s_{3} s_{3}^{(2 \kappa+1) 2+c \varphi(4)-2} \int_{0}^{1} \int_{0}^{1} d t_{2} d t_{4} t_{2}^{c \alpha_{2}}\left(1+t_{2}\right)^{c \alpha_{3}}\left(1+t_{2}+t_{4}\right)^{c \alpha_{4}}\left(1+t_{4}\right)^{c \alpha_{3}} t_{4}^{c \alpha_{2}},
$$

which is bounded in the argument above as

$$
I_{3}(4) \leq C \int_{0}^{1} d s_{3} s_{3}^{\zeta(4)-2} \int_{0}^{1} d t_{2} t_{2}^{c \alpha_{2}+2 \kappa-1} \int_{0}^{1} d t_{4} t_{4}^{c \alpha_{2}+2 \kappa-1} .
$$

(Note throughout that $2 \kappa-1<0$ and $\alpha_{k} \leq 0$.) Hence,

$$
I_{n}(2 p) \leq C \int_{0}^{1} d s_{n} s_{n}^{\zeta(2 p)-2} I(n-1) I(2 p-(n-1)),
$$

which is bounded by induction, and since $\zeta(2 p)>1$. The case of odd $q=2 p-1$ can be considered similarly and involves the integral

$$
\int_{0}^{1} d s_{n} s_{n}^{(2 \kappa-1)(p-1)+2 p-3+c \varphi(2 p-1)} .
$$

This integral is finite since $(2 \kappa-1)(p-1)+2 p-3+c \varphi(2 p-1)>(\kappa+1 / 2)(2 p-1)+c \varphi(2 p-1)-1>0$.

Case $(B)$ : We will show that $r^{-(c \varphi(2)+2 \kappa) p} J_{r}(p) \sim C$, where

$$
J_{r}(p)=\int_{0}^{1} \ldots \int_{0}^{1} d w_{2} \ldots d w_{2 p} \exp \left\{-\sum_{2 \leq k \leq l \leq 2 p} \alpha_{l-k+2} m_{r}\left(w_{k}+\ldots+w_{l}\right)\right\} \prod_{k=1}^{p} w_{2 k}^{2 \kappa-1} .
$$

This is one of the terms in (5.7) with $\{(i, j)\}=\{(1,2),(3,4), \ldots,(2 p-1,2 p)\}$. We will argue that the terms in (5.7) with other $\{(i, j)\} \in \mathcal{P}_{2 p}$ have slower rate of growth than $r^{(c \varphi(2)+2 \kappa) p}$.

Let $\widetilde{J}_{r}(p)$ be the integral $J_{r}(p)$ restricted to $w_{2}>r, \ldots, w_{2 p}>r$. We will show first that $\widetilde{J}_{r}(p) \sim r^{(c \varphi(2)+2 \kappa) p} C$ and then argue that the same happens with $J_{r}(p)$. Observe that

$$
\widetilde{J}_{r}(p)=\int_{0}^{1} \ldots \int_{0}^{1} d w_{2} \ldots d w_{2 p} \prod_{2 \leq k \leq l \leq 2 p}\left(w_{k}+\ldots+w_{l}\right)^{c \alpha_{l-k+2}} \prod_{k=1}^{p} w_{2 k}^{2 \kappa-1} .
$$

Making a change of variables $w_{2 k}=r v_{2 k}, k=1, \ldots, p, w_{2 k+1}=u_{2 k+1}, k=1, \ldots, p-1$, we obtain that

$$
\begin{gathered}
\widetilde{J}_{r}(p)=r^{\left(c \alpha_{2}+2 \kappa\right) p} \int_{1}^{r^{-1}} \int_{1}^{r^{-1}} \ldots \int_{1}^{r^{-1}} d v_{2} d v_{4} \ldots d v_{2 p} \prod_{k=1}^{p} v_{2 k}^{c \alpha_{2}+2 \kappa-1} \\
\int_{r}^{1} \int_{r}^{1} \ldots \int_{r}^{1} d u_{3} d u_{5} \ldots d u_{2 p-1} \prod_{2 \leq k \leq l \leq 2 p}\left(u_{k}+\ldots+u_{l}\right)^{c \alpha_{l-k+2}}
\end{gathered}
$$

where $u_{2 k}=r v_{2 k}$ by convention. Then, by dominated convergence theorem,

$$
\widetilde{J}_{r}(p) \sim r^{(c \varphi(2)+2 \kappa) p}\left(\int_{1}^{\infty} d v v^{c \varphi(2)+2 \kappa-1}\right)^{p} L(p),
$$

as long as $L(p)<\infty$ (recall also that $c \varphi(2)+2 \kappa<0)$, where

$$
L(p)=\int_{0}^{1} \int_{0}^{1} \ldots \int_{0}^{1} d u_{3} d u_{5} \ldots d u_{2 p-1} \prod_{1 \leq k<l \leq p-1}\left(u_{2 k+1}+\ldots+u_{2 l+1}\right)^{c \beta_{l-k}}
$$


and

$$
\beta_{m}=\alpha_{2 m}+2 \alpha_{2 m+1}+\alpha_{2 m+2}, \quad m=1, \ldots, p-1 .
$$

Showing that $L(p)<\infty$ can be done by induction in $p$, similarly to and easier than in case $(A)$ above, if

$$
c \sum_{1 \leq k<l \leq q} \beta_{l-k}+(q-1)+1>0, \quad q=2, \ldots, p-1 .
$$

Observe that $\sum_{1 \leq k<l \leq q} \beta_{l-k}=\sum_{2 \leq k \leq l \leq 2 q} \alpha_{l-k+2}-(q+1) \alpha_{2}=\varphi(2 q+2)-(q+1) \varphi(2)$. Hence, the condition above becomes

$$
(1-c \varphi(2)) \frac{2 q}{2}+c \varphi(2 q)>1, \quad q=3, \ldots, p .
$$

Since $(1-c \varphi(2))+c \varphi(2) \geq 1$ and $\varphi$ is concave, these conditions just follow from $(1-c \varphi(2)) \frac{2 p}{2}+$ $c \varphi(2 p)>1$.

The integral $J_{r}(p)$ restricted to other regions than that in $\widetilde{J}_{r}(p)$ can be dealt with in a similar way. For example, the integral $J_{r}(p)$ over $w_{2}<r, w_{4}<r, \ldots, w_{2 p}<r$, and $w_{3}>r, w_{5}>r, \ldots, w_{2 p-1}>r$ can be dealt with in exactly the same way as $\widetilde{J}_{r}(p)$, and has the rate of growth $r^{(c \varphi(2)+2 \kappa) p}$. The integral $J_{r}(p)$ over $w_{2}<r, w_{4}<r, \ldots, w_{2 p}<r$, and $w_{3}<r, w_{5}>r, \ldots, w_{2 p-1}>r$ has the rate of growth $o\left(r^{(c \varphi(2)+2 \kappa) p}\right)$ because of the restriction $w_{3}<r$.

Finally, consider the terms in (5.7) with $\{(i, j)\} \in \mathcal{P}_{2 p}$ other than $\{(i, j)\}=\{(1,2),(3,4), \ldots,(2 p-$ $1,2 p)\}$. These terms can be shown to be of the order $o\left(r^{(c \varphi(2)+2 \kappa) p}\right)$. The basic idea is the following. (We will omit further details for shortness sake.) For example, such term over $w_{k}>r, k=2, \ldots, 2 p$, can be bounded by

$$
\int_{r}^{1} \ldots \int_{r}^{1} d w_{2} \ldots d w_{2 p} \prod_{2 \leq k \leq l \leq 2 p}\left(w_{k}+\ldots+w_{l}\right)^{c \widetilde{\alpha}_{l-k+2}} \prod_{\left\{k^{\prime}\right\}} w_{k^{\prime}}^{\kappa+\kappa^{\prime}-1},
$$

where $\widetilde{\alpha}_{2}=\alpha_{2}=\varphi(2), \widetilde{\alpha}_{m}$ 's satisfy the assumptions of $\alpha_{m}$ 's, there are $p$ different $k^{\prime}$ such that $\left\{k^{\prime}\right\} \subset\{2, \ldots, 2 p\}$, and $\kappa^{\prime}<\kappa$. For example, in case $p=2$, the integral

$$
\begin{gathered}
\int_{r}^{1} \int_{r}^{1} \int_{r}^{1} d w_{2} d w_{3} d w_{4} w_{2}^{c \alpha_{2}}\left(w_{2}+w_{3}\right)^{c \alpha_{3}}\left(w_{2}+w_{3}+w_{4}\right)^{c \alpha_{4}} . \\
\cdot w_{3}^{c \alpha_{2}}\left(w_{3}+w_{4}\right)^{c \alpha_{3}} w_{4}^{c \alpha_{2}}\left(w_{2}+w_{3}\right)^{2 \kappa-1}\left(w_{3}+w_{4}\right)^{2 \kappa-1}
\end{gathered}
$$

is bounded by

$$
\begin{gathered}
\int_{r}^{1} \int_{r}^{1} \int_{r}^{1} d w_{2} d w_{3} d w_{4} w_{2}^{c \alpha_{2}+\kappa+\kappa^{\prime}-1}\left(w_{2}+w_{3}\right)^{c \alpha_{3}+\left(\kappa-\kappa^{\prime}\right)}\left(w_{2}+w_{3}+w_{4}\right)^{c \alpha_{4}} \\
\cdot w_{3}^{c \alpha_{2}}\left(w_{3}+w_{4}\right)^{c \alpha_{3}+\left(\kappa-\kappa^{\prime}\right)} w_{4}^{c \alpha_{2}+\kappa+\kappa^{\prime}-1}
\end{gathered}
$$

When $\kappa^{\prime}$ is close enough to $\kappa, \kappa^{\prime}<\kappa$, the exponents $\widetilde{\alpha}_{2}=\alpha_{2}, \widetilde{\alpha}_{3}=\alpha_{3}+\kappa-\kappa^{\prime}, \widetilde{\alpha}_{4}=\alpha_{4}$ have properties of the original $\alpha_{2}, \alpha_{3}, \alpha_{4}$. Because of the presence of $\kappa^{\prime}<\kappa$, an argument similar to that above would show that $(5.14)$ is of the order $o\left(r^{(c \varphi(2)+2 \kappa) p}\right)$.

Proof of Proposition 3.2: We consider the simpler case of the exact scale invariance (2.15), and only $t=1$. Observe that, by conditioning on $Q_{r}$ and using (2.26) and (2.32),

$$
\frac{E Z_{r}^{\kappa}(1)^{2}}{C_{\kappa} \Gamma(\kappa+1)^{2}}=E \int_{0}^{1}\left\{s^{-\kappa} \kappa \int_{s}^{1}\left(u^{\kappa} Q_{r}(u)-s^{\kappa} Q_{r}(s)\right)(u-s)^{\kappa-1} d u+Q_{r}(s)(1-s)^{\kappa}\right\}^{2} d s
$$




$$
\begin{gathered}
=\kappa^{2} E \int_{0}^{1} s^{-2 \kappa}\left\{\int_{s}^{1}\left(u^{\kappa} Q_{r}(u)-s^{\kappa} Q_{r}(s)\right)(u-s)^{\kappa-1} d u\right\}^{2} d s \\
+2 \kappa E \int_{0}^{1} s^{-\kappa} Q_{r}(s)(1-s)^{\kappa}\left\{\int_{s}^{1}\left(u^{\kappa} Q_{r}(u)-s^{\kappa} Q_{r}(s)\right)(u-s)^{\kappa-1} d u\right\} d s \\
+E \int_{0}^{1} Q_{r}(s)^{2}(1-s)^{2 \kappa} d s=: \kappa^{2} T_{1, r}+2 \kappa T_{2, r}+T_{3, r} .
\end{gathered}
$$

To prove (3.4), we will show that $T_{1, r}$ diverges faster than $T_{2, r}$ and $T_{3, r}$, and find the corresponding rate of divergence of $T_{1, r}$. We first examine $T_{3, r}$ and $T_{2, r}$, and then turn to $T_{1, r}$.

The case of $T_{3, r}$ is simple. By using (2.9) and (5.2), we have

$$
T_{3, r}=e^{-c \varphi(2)} r^{c \varphi(2)} \int_{0}^{1}(1-s)^{2 \kappa} d s=\frac{e^{-c \varphi(2)} r^{c \varphi(2)}}{2 \kappa+1}=O\left(r^{c \varphi(2)}\right) .
$$

Turning to $T_{2, r}$, we have

$$
\begin{gathered}
T_{2, r}=\int_{0<s<u<1}\left(1_{\{u-s>r\}}+1_{\{u-s<r\}}\right) s^{-\kappa}(1-s)^{\kappa} . \\
\cdot\left(u^{\kappa} E Q_{r}(u) Q_{r}(s)-s^{\kappa} E Q_{r}(s)^{2}\right)(u-s)^{\kappa-1} d s d u=: T_{2, r}^{(1)}+T_{2, r}^{(2)} .
\end{gathered}
$$

By using (2.10) and (5.2),

$$
\begin{aligned}
T_{2, r}^{(1)} & =\int_{0<s<u<1, u-s>r} s^{-\kappa}(1-s)^{\kappa}\left(u^{\kappa}(u-s)^{c \varphi(2)}-s^{\kappa} e^{-c \varphi(2)} r^{c \varphi(2)}\right)(u-s)^{\kappa-1} d u d s \\
& =I_{1}-r^{c \varphi(2)} e^{-c \varphi(2)} I_{2}
\end{aligned}
$$

and, since $\kappa<0$,

$$
\begin{aligned}
I_{1} & =\int_{0<s<u<1, u-s>r} s^{-\kappa}(1-s)^{\kappa} u^{\kappa}(u-s)^{c \varphi(2)+\kappa-1} d u d s \\
& \leq \int_{0<s<u<1, u-s>r}(1-s)^{\kappa}(u-s)^{c \varphi(2)+\kappa-1} d u d s \\
& =\frac{1}{c \varphi(2)+\kappa}\left(\frac{1-r^{c \varphi(2)+2 \kappa+1}}{c \varphi(2)+2 \kappa+1}-r^{c \varphi(2)+\kappa} \frac{1-r^{\kappa+1}}{\kappa+1}\right)=O\left(r^{c \varphi(2)+\kappa}\right),
\end{aligned}
$$

and

$$
I_{2}=\int_{0<s<u<1, u-s>r}(1-s)^{\kappa}(u-s)^{\kappa-1} d u d s=\frac{1}{\kappa}\left(\frac{1-r^{2 \kappa+1}}{2 \kappa+1}-\frac{r^{\kappa}\left(1-r^{\kappa+1}\right)}{\kappa+1}\right)=O\left(r^{\kappa}\right) .
$$

Thus, by collecting the above expressions,

$$
T_{2, r}^{(1)}=O\left(r^{c \varphi(2)+\kappa}\right)
$$

By using (2.10) and (5.2),

$$
\begin{aligned}
T_{2, r}^{(2)} & =e^{-c \varphi(2)} r^{c \varphi(2)} \int_{0<s<u<1, u-s<r} s^{-\kappa}(1-s)^{\kappa}\left(u^{\kappa} e^{c \varphi(2) \frac{u-s}{r}}-s^{\kappa}\right)(u-s)^{\kappa-1} d s d u \\
& =-e^{-c \varphi(2)} r^{c \varphi(2)}\left(J_{1}+J_{2}\right) .
\end{aligned}
$$


Here,

$$
\begin{aligned}
J_{1} & =\int_{0<s<u<1, u-s<r} s^{-\kappa}(1-s)^{\kappa}\left(s^{\kappa}-u^{\kappa}\right) e^{c \varphi(2) \frac{u-s}{r}}(u-s)^{\kappa-1} d s d u \\
& \leq \int_{0}^{r} d w w^{\kappa-1} \int_{0}^{1-w} s^{-\kappa}(1-s)^{\kappa}\left(s^{\kappa}-(s+w)^{\kappa}\right) d s .
\end{aligned}
$$

Observe that, with $\varepsilon<1$,

$$
0 \leq s^{\kappa}-(s+w)^{\kappa}=-\kappa \int_{s}^{s+w} u^{\kappa-1} d u \leq-\kappa s^{\kappa-1+\varepsilon} \int_{s}^{s+w} u^{-\varepsilon} d u \leq-\frac{\kappa}{1-\varepsilon} s^{\kappa-1+\varepsilon} w^{1-\varepsilon} .
$$

Hence, with $\kappa+1<\varepsilon<1$,

$$
J_{1} \leq-\frac{\kappa}{1-\varepsilon} \int_{0}^{r} w^{\kappa-\varepsilon} d w \int_{0}^{1} s^{-1+\varepsilon}(1-s)^{\kappa} d s=O\left(r^{\kappa-\varepsilon+1}\right) .
$$

Similarly,

$$
\begin{aligned}
J_{2} & =\int_{0<s<u<1, u-s<r}(1-s)^{\kappa} \kappa\left(1-e^{c \varphi(2) \frac{u-s}{r}}\right)(u-s)^{\kappa-1} d s d u \\
& \leq-\frac{c \varphi(2)}{r} \int_{0<s<u<1, u-s<r}(1-s)^{\kappa}(u-s)^{\kappa} d u d s \leq-\frac{c \varphi(2)}{(\kappa+1)^{2}} r^{\kappa}=O\left(r^{\kappa}\right) .
\end{aligned}
$$

Collecting the above results yields

$$
T_{2, r}^{(2)}=O\left(r^{c \varphi(2)+\kappa}\right)
$$

and the relations (5.17), (5.18) and (5.19) show that

$$
T_{2, r}=O\left(r^{c \varphi(2)+\kappa}\right) .
$$

Consider now the term $E T_{1, r}$ in (5.15). Let

$$
F(u)=\left\{\begin{array}{cc}
|u|^{c \varphi(2)} & \text { if }|u|>1 \\
e^{-c \varphi(2)} e^{c \varphi(2)|u|} & \text { if }|u|<1
\end{array}\right.
$$

By making a change of variables $s=r z, u-s=r x, v-s=r y$ and using (2.10) and (5.2), we have

$$
\begin{gathered}
\frac{T_{1, r}}{2}=\int_{0<s<u<v<1} s^{-2 \kappa}\left(u^{\kappa} v^{\kappa} E Q_{r}(u) Q_{r}(v)-u^{\kappa} s^{\kappa} E Q_{r}(u) Q_{r}(s)\right. \\
\left.-v^{\kappa} s^{\kappa} E Q_{r}(v) Q_{r}(s)+s^{2 \kappa} E Q_{r}(s)^{2}\right)(u-s)^{\kappa-1}(v-s)^{\kappa-1} d s d u d v \\
=r^{2 \kappa+c \varphi(2)} \int_{0}^{r^{-1}} d z \int_{0}^{r^{-1}-z} d x \int_{x}^{r^{-1}-z} d y\left(\left(1+\frac{x}{z}\right)^{\kappa}\left(1+\frac{y}{z}\right)^{\kappa} F(y-x)\right. \\
\left.-\left(1+\frac{x}{z}\right)^{\kappa} F(x)-\left(1+\frac{y}{z}\right)^{\kappa} F(y)+F(0)\right) x^{\kappa-1} y^{\kappa-1} \\
\sim r^{2 \kappa+c \varphi(2)} \int_{0<x<y}(F(y-x)-F(x)-F(y)+F(0)) x^{\kappa-1} y^{\kappa-1} d x d y .
\end{gathered}
$$

The result (3.4) follows from (5.16), (5.20) and (5.21), since $\kappa<0$.

Proof of Lemma 3.1: In the case $\kappa \in(0,1 / 2)$,

$$
E Z_{r}^{\kappa}(t) Z_{r^{*}}^{\kappa}(t)=C_{\kappa} \int_{0}^{t} \int_{0}^{t} E Q_{r}(u) Q_{r^{*}}(v)|u-v|^{2 \kappa-1} d u d v
$$




$$
=C_{\kappa} \int_{0}^{t} \int_{0}^{t} E Q_{r}(u) Q_{r}(v)|u-v|^{2 \kappa-1} d u d v=E Z_{r}^{\kappa}(t)^{2},
$$

since $\left\{Q_{r}(v)\right\}_{r>0}$ is a martingale (Lemma 3 in Chainais et al. (2005a)). The case $\kappa \in(-1 / 2,0)$ can be proved in a similar way using the expression of the type (5.15).

Proof of Theorem 3.3: Using Theorem 4.2.3 in Kwapień and Woyczyński (1992), it is enough to show that:

(i) $\left\{Z_{1 / T}^{\kappa}(t)\right\}_{t \in[0,1]}$ is a martingale in $T \geq 1$.

(ii) $\left\{Z_{1 / T}^{\kappa}(t)\right\}_{t \in[0,1]}$ converges in distribution.

(iii) $\sup _{T \geq 1} E \sup _{t \in[0,1]}\left|Z_{1 / T}^{\kappa}(t)\right|<\infty$.

The martingale property in $(i)$ is equivalent to $\left\{Z_{1 / T}^{\kappa}(t), T \geq 1\right\}$ being a martingale for fixed $t$ (but with respect to the same filtration for all $t)$. The proof of $(i)-(i i i)$ is given next.

(i) Consider a filtration $\mathcal{F}=\left\{\mathcal{F}_{T}, T \geq 1\right\}$ of $\sigma$-fields given by $\mathcal{F}_{T}=\sigma\{M(A), A \in \mathcal{B}([1 / T, \infty) \times$ $\mathbb{R})\} \vee \sigma\left\{B^{\kappa}(t), t \in \mathbb{R}\right\}$, where $\mathcal{B}$ indicates Borel $\sigma$-field. The $\sigma$-field $\mathcal{F}_{S}$ is generated by smooth variables of the form

$$
\Phi=F\left(M\left(A_{1}\right), \ldots, M\left(A_{n}\right)\right) \exp \left\{i \sum_{j=1}^{m} \beta_{j} B^{\kappa}\left(t_{j}\right)\right\},
$$

where $m, n \in \mathbb{N} \cup\{0\}, F: \mathbb{R}^{n} \mapsto \mathbb{R}$ is a bounded, continuous function, $\beta_{j}, t_{j} \in \mathbb{R}$, and $A_{j} \in$ $\mathcal{B}([1 / S, \infty) \times \mathbb{R})$. For the martingale property, it is then enough to show that $E \Phi Z_{1 / T}^{\kappa}(t)=E \Phi Z_{1 / S}^{\kappa}(t)$ for $T \geq S \geq 1$.

Letting $E^{M}$ be the expectation conditionally on $M$, observe that $\left(Z_{1 / T}^{\kappa}(t), B^{\kappa}\left(t_{1}\right), \ldots, B^{\kappa}\left(t_{m}\right)\right)$ is conditionally Gaussian vector with the covariance structure consisting of, in particular,

$$
E^{M} Z_{1 / T}^{\kappa}(t) B^{\kappa}\left(t_{j}\right)=C_{\kappa} \int_{0}^{t} \int_{0}^{t_{j}} Q_{1 / T}(u)|u-v|^{2 \kappa-1} d u d v .
$$

Using the fact that, for a Gaussian vector $\left(X_{1}, \ldots, X_{m+1}\right)$ with covariance matrix $\left(c_{k, l}\right)_{k, l=1 \ldots, m+1}$,

$$
E X_{1} \exp \left\{i \sum_{j=2}^{m+1} \beta_{j} X_{j}\right\}=-i \sum_{j=2}^{m+1} \beta_{j} c_{1, j} E \exp \left\{i \sum_{j=2}^{m+1} \beta_{j} X_{j}\right\},
$$

this yields that

$$
\begin{gathered}
E \Phi Z_{1 / T}^{\kappa}(t)=-i C_{\kappa} \sum_{j=1}^{m} E\left(F\left(M\left(A_{1}\right), \ldots, M\left(A_{n}\right)\right) .\right. \\
\left.\int_{0}^{t} \int_{0}^{t_{j}} Q_{1 / T}(u)|u-v|^{2 \kappa-1} d u d v \exp \left\{i \sum_{j=1}^{m} \beta_{j} B^{\kappa}\left(t_{j}\right)\right\}\right) .
\end{gathered}
$$

Using the martingale property of $\left\{Q_{1 / T}(u), T \geq 1\right\}$ and conditioning on $\mathcal{F}_{S}$, the last expression is equal to $E \Phi Z_{1 / S}^{\kappa}(t)$.

(ii) By Proposition 3.1, the collection $\left\{Z_{1 / T}^{\kappa}(t), T \geq 1\right\}$ is bounded in $L^{2}(\Omega)$. Then, since $\left\{Z_{1 / T}^{\kappa}(t), T \geq 1\right\}$ is a martingale by $(i)$, it converges almost surely. The almost sure convergence also implies that of finite dimensional distributions. For the convergence in distribution in the space of continuous functions $C([0,1], \mathbb{R})$, since $c \varphi(2)+2 \kappa>0$, it is therefore enough to show (Billingsley (1968)) that, for all $T \geq 1,0 \leq t_{1} \leq t_{2} \leq 1$,

$$
E\left(Z_{1 / T}^{\kappa}\left(t_{2}\right)-Z_{1 / T}^{\kappa}\left(t_{1}\right)\right)^{2} \leq C\left(t_{2}-t_{1}\right)^{c \varphi(2)+2 \kappa+1} .
$$


For this, use the fact $E Q_{1 / T}(u) Q_{1 / T}(v) \leq C|u-v|^{c \varphi(2)}$ for all $T \geq 1$, to write

$$
E\left(Z_{1 / T}^{\kappa}\left(t_{2}\right)-Z_{1 / T}^{\kappa}\left(t_{1}\right)\right)^{2} \leq c \int_{t_{1}}^{t_{2}} \int_{t_{1}}^{t_{2}}|u-v|^{c \varphi(2)+2 \kappa-1} d u d v=c^{\prime}\left(t_{2}-t_{1}\right)^{c \varphi(2)+2 \kappa+1} .
$$

(iii) For this part, observe that

$$
E \sup _{t \in[0,1]}\left|Z_{1 / T}^{\kappa}(t)\right|=\int_{0}^{\infty} P\left(\sup _{t \in[0,1]}\left|Z_{1 / T}^{\kappa}(t)\right|>x\right) d x=E \int_{0}^{\infty} P\left(\sup _{t \in[0,1]}\left|Z_{1 / T}^{\kappa}(t)\right|>x \mid Q_{1 / T}\right) d x .
$$

Conditionally on $Q_{1 / T}, Z_{1 / T}^{\kappa}(t)$ is a Gaussian continuous process in $t$. By the Borell inequality for continuous Gaussian processes (Adler (1990), relation (2.6) on p. 43), we have

$$
P\left(\sup _{t \in[0,1]}\left|Z_{1 / T}^{\kappa}(t)\right|>x \mid Q_{1 / T}\right) \leq 2 \exp \left\{-\frac{1}{2}\left(x-E\left(\sup _{t \in[0,1]}\left|Z_{1 / T}^{\kappa}(t)\right| \mid Q_{1 / T}\right)\right)^{2} / \sigma_{T}^{2}\right\},
$$

where

$$
\sigma_{T}^{2}=\sup _{t \in[0,1]} E\left(\left|Z_{1 / T}^{\kappa}(t)\right|^{2} \mid Q_{1 / T}\right)
$$

Substituting (5.23) into (5.22), extending the integration range to $\mathbb{R}$ and making a change of variables $x-E\left(\sup _{t \in[0,1]} \mid Z_{1 / T}^{\kappa}(t) \| Q_{1 / T}\right)=y$ yields

$$
E \sup _{t \in[0,1]}\left|Z_{1 / T}^{\kappa}(t)\right| \leq 2 E \int_{\mathbb{R}} e^{-\frac{y^{2}}{2 \sigma_{T}^{2}}} d y=2 \sqrt{2 \pi} E \sigma_{T} \leq 2 \sqrt{2 \pi}\left(E \sigma_{T}^{2}\right)^{1 / 2}
$$

Since $Q_{1 / T}(u)>0$, observe now that

$$
\sigma_{T}^{2}=C_{\kappa} \sup _{t \in[0,1]} \int_{0}^{t} \int_{0}^{t} Q_{1 / T}(u) Q_{1 / T}(v)|u-v|^{2 \kappa-1} d u d v=C_{\kappa} \int_{0}^{1} \int_{0}^{1} Q_{1 / T}(u) Q_{1 / T}(v)|u-v|^{2 \kappa-1} d u d v .
$$

Hence, $E \sigma_{T}^{2}=E Z_{1 / T}^{\kappa}(1)^{2}$ and substituting this into (5.24), we obtain that

$$
\sup _{T \geq 1} E \sup _{t \in[0,1]}\left|Z_{1 / T}^{\kappa}(t)\right| \leq 2 \sqrt{2 \pi}\left(\sup _{T \geq 1} E Z_{1 / T}^{\kappa}(1)^{2}\right)^{1 / 2} .
$$

The conclusion follows since $\sup _{T \geq 1} E Z_{1 / T}^{\kappa}(1)^{2}<\infty$ by Proposition 3.1.

Proof of Theorem 3.4: We first show (3.13) and work with $t=1$, for simplicity. Observe that, for $k \in \mathbb{N}$,

$$
E\left|Z_{r}^{\kappa}(1)\right|^{q}=C_{\kappa, q} E\left(\int_{0}^{1} \int_{0}^{1} Q_{r}(u) Q_{r}(v)|u-v|^{2 \kappa-1} d u d v\right)^{q / 2} \leq C_{\kappa, q}\left(I_{1}+I_{2}\right),
$$

where

$$
\begin{aligned}
& I_{1}=E\left(\int_{0}^{1} \int_{0}^{1} 1_{\left\{|u-v| \leq k^{-1} / 2\right\}} Q_{r}(u) Q_{r}(v)|u-v|^{2 \kappa-1} d u d v\right)^{q / 2}, \\
& I_{2}=E\left(\int_{0}^{1} \int_{0}^{1} 1_{\left\{|u-v|>k^{-1} / 2\right\}} Q_{r}(u) Q_{r}(v)|u-v|^{2 \kappa-1} d u d v\right)^{q / 2} .
\end{aligned}
$$

By a change of variables $u=k x, v=k y$ and relations (2.16) and (2.17), we get that

$$
I_{1}=k^{-\zeta(q)} E\left(\int_{0}^{k} \int_{0}^{k} 1_{\{|x-y| \leq 1 / 2\}} Q_{k r}(x) Q_{k r}(y)|x-y|^{2 \kappa-1} d x d y\right)^{q / 2},
$$


where $r$ is such that $r \leq k^{-1}$. Note further that, by stationarity of $Q_{k r}(\cdot)$,

$$
\begin{gathered}
k^{\zeta(q)} I_{1} \leq \sum_{n=0}^{2 k-1} E\left(\int_{n / 2}^{(n+1) / 2} \int_{n / 2}^{(n+1) / 2} 1_{\{|x-y| \leq 1 / 2\}} Q_{k r}(x) Q_{k r}(y)|x-y|^{2 \kappa-1} d x d y\right)^{q / 2} \\
+2 \sum_{n=0}^{2 k-2} E\left(\int_{n / 2}^{(n+1) / 2} \int_{(n+1) / 2}^{(n+2) / 2} 1_{\{|x-y| \leq 1 / 2\}} Q_{k r}(x) Q_{k r}(y)|x-y|^{2 \kappa-1} d x d y\right)^{q / 2} \\
\quad=2 k E\left(\int_{0}^{1 / 2} \int_{0}^{1 / 2} 1_{\{|x-y| \leq 1 / 2\}} Q_{k r}(x) Q_{k r}(y)|x-y|^{2 \kappa-1} d x d y\right)^{q / 2} \\
+2(2 k-1) E\left(\int_{0}^{1 / 2} \int_{1 / 2}^{1} 1_{\{|x-y| \leq 1 / 2\}} Q_{k r}(x) Q_{k r}(y)|x-y|^{2 \kappa-1} d x d y\right)^{q / 2} \\
\leq 6 k E\left(\int_{0}^{1} \int_{0}^{1} 1_{\{|x-y| \leq 1 / 2\}} Q_{k r}(x) Q_{k r}(y)|x-y|^{2 \kappa-1} d x d y\right)^{q / 2}=\frac{6 k}{C_{\kappa, q}} E\left|Z_{k r}^{\kappa}(1)\right|^{q} .
\end{gathered}
$$

Since $\left\{Z_{1 / s}^{\kappa}(1)\right\}_{s \geq 1}$ is a martingale (see the proof of Theorem 3.3) and $r \leq r k$,

$$
I_{1} \leq \frac{6 k^{1-\zeta(q)}}{C_{\kappa, q}} E\left|Z_{r}^{\kappa}(1)\right|^{q}
$$

On the other hand,

$$
I_{2} \leq(2 k)^{(1-\kappa / 2) q} E\left(\int_{0}^{1} Q_{r}(u) d u\right)^{q} .
$$

If $k$ is such that $6 k^{1-\zeta(q)}<1$, we get from (5.25), (5.26) and (5.27) that

$$
E\left|Z_{r}^{\kappa}(1)\right|^{q} \leq \frac{(2 k)^{(1-\kappa / 2) q}}{1-6 k^{1-\zeta(q)}} E\left(\int_{0}^{1} Q_{r}(u) d u\right)^{q}
$$

for all $r \leq k^{-1}$. Since $q+c \varphi(q)>\left(\kappa_{c}+1 / 2\right) q+c \varphi(q)=\zeta(q)>1$, Lemma 3, (i), in Bacry and Muzy (2003) yields that $\sup _{r \in(0,1]} E\left(\int_{0}^{1} Q_{r}(u) d u\right)^{q}<\infty$. This and (5.28) show that, with $k$ as above,

$$
\sup _{r \in(0,1]} E\left|Z_{r}^{\kappa}(1)\right|^{q} \leq \sup _{r \in\left(0, k^{-1}\right]} E\left|Z_{r}^{\kappa}(1)\right|^{q}<\infty
$$

which is (3.13). The last statement of the theorem follows from (3.13) and the fact that $\left\{Z_{1 / s}^{\kappa}(1)\right\}_{s \geq 1}$ is a martingale.

Proof of Proposition 4.1: The relation (4.1) can be shown as in the proof of Proposition 3.1. To see (4.2), observe that, for $k>1 / \tau+1$ and using (4.1),

$$
\begin{aligned}
& E X_{k} X_{0}=C_{\kappa} \int_{k \tau}^{(k+1) \tau} \int_{0}^{\tau} E Q_{0}(u) Q_{0}(v)|u-v|^{2 \kappa-1} d u d v=C_{\kappa} \int_{k \tau}^{(k+1) \tau} \int_{0}^{\tau}|u-v|^{2 \kappa-1} d u d v \\
& =C_{\kappa} \tau^{2 \kappa+1} \int_{k}^{k+1} \int_{0}^{1}|u-v|^{2 \kappa-1} d u d v=\frac{C_{\kappa} \tau^{2 \kappa+1}}{2 \kappa(2 \kappa+1)}\left(|k+1|^{2 \kappa+1}+|k-1|^{2 \kappa+1}-2|k|^{2 \kappa+1}\right) .
\end{aligned}
$$

A proof of the relation (4.3) depends on various cases involving $s$ and $t$. For example, consider the case when $s>1$ and $t-s>1$. Then,

$$
C_{\kappa}^{-1} E Z_{r}^{\kappa}(s) Z_{r}^{\kappa}(t)=\int_{0}^{s} \int_{0}^{t} E Q_{r}(u) Q_{r}(v)|u-v|^{2 \kappa-1} d u d v=: J_{1, r}+J_{2, r}+J_{3, r}
$$


where $J_{1, r}, J_{2, r}$ and $J_{3, r}$ are the integrals restricted to $|u-v|>1, r<|u-v|<1$ and $|u-v|<r$, respectively. Observe that $J_{1, r}=\int_{0}^{s} \int_{0}^{t}|u-v|^{2 \kappa-1} 1_{\{|u-v|>1\}} d u d v<\infty$. The term $J_{2, r}$ can be written as

$$
\begin{aligned}
& J_{2, r}=\int_{0}^{s} d u \int_{u+r}^{u+1} d v(v-u)^{c \varphi(2)+2 \kappa-1}+\left\{\int_{r}^{1} d u \int_{0}^{u-r} d v+\int_{1}^{s} d u \int_{u-1}^{u-r} d v\right\}(u-v)^{c \varphi(2)+2 \kappa-1} \\
= & s \int_{r}^{1} d z z^{c \varphi(2)+2 \kappa-1}+\left\{\int_{0}^{1} d u \int_{r}^{u} d z+\int_{1}^{s} d u \int_{r}^{1} d z\right\} z^{c \varphi(2)+2 \kappa-1} \sim r^{c \varphi(2)+2 \kappa} 2 s \int_{1}^{\infty} d w w^{c \varphi(2)+2 \kappa-1} .
\end{aligned}
$$

One can similarly show that $I_{3, r} \sim r^{c \varphi(2)+2 \kappa} 2 s e^{-c \varphi(2)} \int_{0}^{1} d w e^{c \varphi(2) w} w^{2 \kappa-1}$, and deal with the other cases of $s$ and $t$.

Proof of Proposition 4.2: The result is immediate from the proof of Proposition 3.1.

Proof of Theorem 4.2: Consider first the case $t=b^{n}, n \geq 1$, with $r<b^{n}<b^{n-1}<\ldots<b<$ 1. Set

$$
Q_{r / b^{n}}^{(n)}(u)=Q_{r}^{b^{n}}\left(b^{n} u\right)
$$

and

$$
Z_{r}^{\kappa, n}(t)=\int_{0}^{t} Q_{r / b^{n}}^{(n)}(u) d B^{\kappa}(u)
$$

Observe that, by conditioning on $Q_{r}(u)$, using (2.24) and (2.7), and making a change of variables $u \leftrightarrow b u, v \leftrightarrow b v$,

$$
\begin{gathered}
E\left|Z_{r}^{\kappa}\left(b^{n}\right)\right|^{q}=C_{\kappa, q} E\left(\int_{0}^{b^{n}} \int_{0}^{b^{n}} Q_{r}(u) Q_{r}(v)|u-v|^{2 \kappa-1} d u d v\right)^{q / 2} \\
=C_{\kappa, q} b^{\left(\kappa+\frac{1}{2}\right) q} E\left(\int_{0}^{b^{n-1}} \int_{0}^{b^{n-1}} Q_{b}(b u) Q_{b}(b v) Q_{r}^{b}(b u) Q_{r}^{b}(b v)|u-v|^{2 \kappa-1} d u d v\right)^{q / 2}
\end{gathered}
$$

and, similarly,

$$
E\left|Z_{r}^{\kappa, 1}\left(b^{n-1}\right)\right|^{q}=C_{\kappa, q} E\left(\int_{0}^{b^{n-1}} \int_{0}^{b^{n-1}} Q_{r}^{b}(b u) Q_{r}^{b}(b v)|u-v|^{2 \kappa-1} d u d v\right)^{q / 2}
$$

Then, by using the inequality (69) in Chainais et al. (2005a) and independence of $\left\{Q_{b}(u)\right\}$ and $\left\{Q_{r}^{b}(u)\right\}$,

$$
\begin{gathered}
\left.|E| Z_{r}^{\kappa}\left(b^{n}\right)\right|^{q}-b^{\left(\kappa+\frac{1}{2}\right) q} E Q_{b}(0)^{q} E\left|Z_{r}^{\kappa, 1}\left(b^{n-1}\right)\right|^{q} \mid \\
\leq C_{\kappa, q} b^{\left(\kappa+\frac{1}{2}\right) q} E \mid\left(\int_{0}^{b^{n-1}} \int_{0}^{b^{n-1}} Q_{b}(b u) Q_{b}(b v) Q_{r}^{b}(b u) Q_{r}^{b}(b v)|u-v|^{2 \kappa-1} d u d v\right)^{q / 2} \\
-Q_{b}(0)^{q}\left(\int_{0}^{b^{n-1}} \int_{0}^{b^{n-1}} Q_{r}^{b}(b u) Q_{r}^{b}(b v)|u-v|^{2 \kappa-1} d u d v\right)^{q / 2} \mid \\
\leq E \sup _{0 \leq u, v \leq b^{n}}\left|Q_{b}(u)^{q / 2} Q_{b}(v)^{q / 2}-Q_{b}(0)^{q}\right| E\left|Z_{r}^{\kappa, 1}\left(b^{n-1}\right)\right|^{q}
\end{gathered}
$$

and this yields

$$
E\left|Z_{r}^{\kappa}\left(b^{n}\right)\right|^{q}=b^{\left(\kappa+\frac{1}{2}\right) q} E Q_{b}(0)^{q} E\left|Z_{r}^{\kappa, 1}\left(b^{n-1}\right)\right|^{q}\left(1+\epsilon_{r, b, n}^{(1)}\right),
$$


where

$$
\left|\epsilon_{r, b, n}^{(1)}\right| \leq\left(E Q_{b}(0)^{q}\right)^{-1} E \sup _{0 \leq u, v \leq b^{n}}\left|Q_{b}(u)^{q / 2} Q_{b}(v)^{q / 2}-Q_{b}(0)^{q}\right|=\tau_{b, q}^{(1)}\left(b^{n-1}\right) .
$$

Focusing now on the term $E\left|Z_{r}^{\kappa, 1}\left(b^{n-1}\right)\right|^{q}$, we have as above

$$
\begin{gathered}
E\left|Z_{r}^{\kappa, 1}\left(b^{n-1}\right)\right|^{q}=C_{\kappa, q} b^{\left(\kappa+\frac{1}{2}\right) q} E\left(\int_{0}^{b^{n-2}} \int_{0}^{b^{n-2}} Q_{b^{2}}^{b}\left(b^{2} u\right) Q_{b^{2}}^{b}\left(b^{2} v\right) Q_{r}^{b^{2}}\left(b^{2} u\right) Q_{r}^{b^{2}}\left(b^{2} v\right)|u-v|^{2 \kappa-1} d u d v\right)^{q / 2}, \\
E\left|Z_{r}^{\kappa, 2}\left(b^{n-2}\right)\right|^{q}=C_{\kappa, q} E\left(\int_{0}^{b^{n-2}} \int_{0}^{b^{n-2}} Q_{r}^{b^{2}}\left(b^{2} u\right) Q_{r}^{b^{2}}\left(b^{2} v\right)|u-v|^{2 \kappa-1} d u d v\right)^{q / 2}
\end{gathered}
$$

and

$$
E\left|Z_{r}^{\kappa, 1}\left(b^{n-1}\right)\right|^{q}=b^{\left(\kappa+\frac{1}{2}\right) q} E Q_{b^{2}}^{b}(0)^{q} E\left|Z_{r}^{\kappa, 2}\left(b^{n-2}\right)\right|^{q}\left(1+\epsilon_{r, b, n}^{(2)}\right),
$$

where

$$
\left|\epsilon_{r, b, n}^{(2)}\right| \leq\left(E Q_{b^{2}}^{b}(0)^{q}\right)^{-1} E \sup _{0 \leq u, v \leq b^{n}}\left|Q_{b^{2}}^{b}(u)^{q / 2} Q_{b^{2}}^{b}(v)^{q / 2}-Q_{b}(0)^{q}\right|=\tau_{b, q}^{(2)}\left(b^{n-2}\right) .
$$

By continuing this way for $E\left|Z_{r}^{\kappa, k}\left(b^{n-k}\right)\right|^{q}, k=2,3, \ldots, n$, and gathering (5.32), (5.33) and similar results for $E\left|Z_{r}^{\kappa, k}\left(b^{n-k}\right)\right|^{q}$, we obtain that

$$
\begin{gathered}
E\left|Z_{r}^{\kappa}\left(b^{n}\right)\right|^{q}=\left(b^{n}\right)^{\left(\kappa+\frac{1}{2}\right) q} E Q_{b}(0)^{q} E Q_{b^{2}}^{b}(0)^{q} \ldots E Q_{b^{n}}^{b^{n-1}}(0)^{q} E\left|Z_{r}^{\kappa, n}(1)\right|^{q} \prod_{k=1}^{n}\left(1+\epsilon_{r, b, n}^{(k)}\right) \\
=\left(b^{n}\right)^{\left(\kappa+\frac{1}{2}\right) q} E Q_{b^{n}}(0)^{q} E\left|Z_{r}^{\kappa, n}(1)\right|^{q} \prod_{k=1}^{n}\left(1+\epsilon_{r, b, n}^{(k)}\right)
\end{gathered}
$$

with

$$
\left|\epsilon_{r, b, n}^{(k)}\right| \leq \tau_{b, q}^{(k)}\left(b^{n-k}\right) .
$$

In the case (2.14), by (2.18), $Q_{r}^{b^{n}}\left(b^{n} u\right)$ has the same finite dimensional distributions as $Q_{r / b^{n}}(u)$ and hence $E\left|Z_{r}^{\kappa, n}(1)\right|^{q}=E\left|Z_{r / b^{n}}^{\kappa}(1)\right|^{q}$. Moreover, $E Q_{b^{n}}(0)^{q}=\left(b^{n}\right)^{c \varphi(q)}$ using (5.1). Together with (5.34), this yields

$$
E\left|\widetilde{Z}_{r}^{\kappa}\left(b^{n}\right)\right|^{q}=\left(b^{n}\right)^{\zeta(q)} E\left|\widetilde{Z}_{r / b^{n}}^{\kappa}(1)\right|^{q} \prod_{k=1}^{n}\left(1+\epsilon_{r, b, n}^{(k)}\right) .
$$

The relation (4.12) for $t=b^{n}$ follows by letting $r \rightarrow 0$ and using the assumptions (4.10) and (4.11). This relation for arbitrary $t \in(0,1]$ follows from that for $t=b^{n}$ by monotonicity.

\section{Acknowledgment}

The authors would like to thank anonymous referees for many useful comments and suggestions.

\section{References}

Adler, R. J. (1990), An introduction to continuity extrema and related topics for general Gaussian processes, in 'IMS Lectures Notes', Institute of Mathematical Statistics, Hayward. Vol. 12.

Bacry, E., Delour, J. \& Muzy, J. (2001), 'Multifractal random walk', Physical Review E 64, 026103.

Bacry, E. \& Muzy, J. (2003), 'Log-infinitely divisible multifractal processes', Communications in Mathematical Physics 236, 449-475. 
Bardet, J.-M., Lang, G., Oppenheim, G., Philippe, A. \& Taqqu, M. S. (2003), Generators of long-range dependent processes: a survey, in P. Doukhan, G. Oppenheim \& M. S. Taqqu, eds, 'Long-Range Dependence: Theory and Applications', Birkhäuser, Boston, pp. 579-623.

Barral, J. \& Mandelbrot, B. B. (2002), 'Multiplicative products of cylindrical pulses', Probability Theory and Related Fields 124, 409-430.

Billingsley, P. (1968), Convergence of Probability Measures, Wiley, New York.

Carmona, P., Coutin, L. \& Montseny, G. (2003), 'Stochastic integration with respect to fractional Brownian motion', Annales de l'Institut Henri Poincaré 39(1), 27-68.

Chainais, P., Riedi, R. \& Abry, P. (2005a), 'On non-scale-invariant infinitely divisible cascades', IEEE Transaction on Information Theory 51(3), 1063-1083.

Chainais, P., Riedi, R. \& Abry, P. (2005b), 'Warped infinitely divisible cascades: beyond scale invariance', Traitement du Signal 22(1), 27-40.

Decreusefond, L. \& Üstünel, A. S. (1999), 'Stochastic analysis of the fractional Brownian motion', Potential Analysis 10, 177-214.

Embrechts, P. \& Maejima, M. (2002), Selfsimilar Processes, Academic Press.

Gripenberg, G. \& Norros, I. (1996), 'On the prediction of fractional Brownian motion', Journal of Applied Probability 33, 400-410.

Jaffard, S. (2004), Wavelet techniques in multifractal analysis, in 'Fractal Geometry and Applications: A Jubilee of Benoit Mandelbrot, M. Lapidus et M. van Frankenhuijsen Eds., Proceedings of Symposia in Pure Mathematics', Vol. 72(2), AMS, pp. 91-152.

Jaffard, S., Lashermes, B. \& Abry, P. (2006), Wavelet leaders in multifractal analysis, in 'Wavelet Analysis and Applications,T Qian, M. I; Vai, X. Yuesheng, Eds.', Birkhäuser Verlag, Basel, Switzerland, pp. 219-264.

Kahane, J.-P. \& Peyriere, J. (1976), 'Sur certaines martingales de Benoit Mandelbrot', Advances in Mathematics 22, 131-145.

Kwapień, S. \& Woyczyński, N. A. (1992), Random Series and Stochastic Integrals: Single and Multiple, Birkhäuser, Boston.

Lashermes, B., Abry, P. \& Chainais, P. (2004), 'New insights into the estimation of scaling exponents', Int. J. of Wavelets, Multiresolution and Information Processing 2(4), 497-523.

Ludeña, C. (2008), ' $L^{p}$-variations for multifractal fractional random walks', The Annals of Applied Probability 18(3), 1138-1163.

Mandelbrot, B. (1999), 'A multifractal walk down Wall Street', Scientific American 280(2), 70-73.

Mandelbrot, B. B. (1974), 'Intermittent turbulence in self-similar cascades; divergence of high moments and dimension of the carrier', Journal of Fluid Mechanics 62, 331-358.

Mannersalo, P., Norros, I. \& Riedi, R. H. (2002), 'Multifractal products of stochastic processes: construction and some basic properties', Advances in Applied Probability 34(4), 888-903.

Muzy, J. \& Bacry, E. (2002), 'Multifractal stationary random measures and multifractal random walks with log-infinitely divisible scaling laws', Physical Review E 66, 056121.

Pipiras, V. \& Taqqu, M. S. (2000), 'Integration questions related to fractional Brownian motion', Probability Theory and Related Fields 118(2), 251-291.

Pipiras, V. \& Taqqu, M. S. (2003), Fractional calculus and its connections to fractional Brownian motion, in P. Doukhan, G. Oppenheim \& M. S. Taqqu, eds, 'Theory and Applications of Long Range Dependence', Birkhäuser, Boston, MA, pp. 165-201.

Riedi, R. (2003), Multifractals processes, in P. Doukhan, G. Oppenheimer \& M. S. Taqqu, eds, 'Theory and Applications of Long Range Dependence', Bilkhäuser, Boston, MA, pp. 625-716.

Samorodnitsky, G. \& Taqqu, M. S. (1994), Stable Non-Gaussian Processes: Stochastic Models with Infinite Variance, Chapman and Hall, New York, London. 
Schertzer, D. \& Lovejoy, S. (1987), 'Physical modeling and analysis of rain and clouds by anisotropic scaling of multiplicative processes', Journal of Geophysical Research D 92(8), 9693-9714.

Schmitt, F. (2003), 'A causal multifractal stochastic equation and its statistical properties', European Physical Journal B 34, 85-98.

Schmitt, F. \& Marsan, D. (2001), 'Stochastic equations generating continuous multiplicative cascades', The European Physical Journal B 20,3-6. 